\title{
NUMERICAL PRECISION FOR DIFFERENTIAL INCLUSIONS WITH UNIQUENESS
}

\author{
JÉRÔMe BAstien ${ }^{1}$ AND Michelle Schatzman ${ }^{2}$
}

\begin{abstract}
In this article, we show the convergence of a class of numerical schemes for certain maximal monotone evolution systems; a by-product of this results is the existence of solutions in cases which had not been previously treated. The order of these schemes is $1 / 2$ in general and 1 when the only non Lipschitz continuous term is the subdifferential of the indicatrix of a closed convex set. In the case of Prandtl's rheological model, our estimates in maximum norm do not depend on spatial dimension.
\end{abstract}

Mathematics Subject Classification. 34A60, 34G25, 34K28, 47H05, 47J35, 65L70.

Received: December 18, 2001.

\section{INTRODUCTION AND SUMMARY}

In $[1,3]$ we studied rheological models involving a dry friction term; the natural model is a system of differential equations with a maximal monotone term and a large number of degrees of freedom; we wrote a numerical method which was implicit with respect to the multivalued term and explicit with respect to everything else, and we found those methods to be experimentally of order 1 with respect to the time step, and we observed that the estimates did not depend on the number of degrees of freedom.

We found very little information in the literature on the order of precision for such methods, with the notable exception of Lippold [16] who obtained a result relative to the order of precision for somewhat simpler systems.

In the foregoing article, we justify the order properties experimentally found, we generalize Lippold's result and a by-product of our analysis is a generalization of some existence and uniqueness result of Brezis [5].

Let $V, H$ and $V^{\prime}$ be three separable Hilbert spaces, equipped with norms and scalar products denoted by $\|\|,.((.,)),.||,.(.,),.\|.\|^{\prime}$ and $((., .))^{\prime}$. We denote by $\langle.,$.$\rangle the duality bracket between V$ and $V^{\prime}$. We assume that these three spaces constitute a Gelfand triple i.e.

$$
V \hookrightarrow H \hookrightarrow V^{\prime},
$$

where we denote by $\hookrightarrow$ a dense and continuous inclusion. $V$ is a dense subspace of $H$. Let $A$ be a maximal monotone operator from $V$ to $V^{\prime}$, with non empty domain $D(A)$; its properties are described in detail in [4-6,18].

\footnotetext{
Keywords and phrases. Differential inclusions, existence and uniqueness, multivalued maximal monotone operator, sub-differential, numerical analysis, implicit Euler numerical scheme, frictions laws.

1 UMR 5585 CNRS, MAPLY, Laboratoire de mathématiques appliquées de Lyon, Université Claude Bernard Lyon I, 69622 Villeurbanne Cedex, France. e-mail: jerome.bastien@utbm.fr

Current address: Laboratoire Mécatronique 3M, Université de Technologie de Belfort-Montbéliard, 90010 Belfort Cedex, France.

2 UMR 5585 CNRS, MAPLY, Laboratoire de mathématiques appliquées de Lyon, Université Claude Bernard Lyon I, 69622 Villeurbanne Cedex, France.
} 
Let $B$ be a Lipschitz continuous and coercive mapping from $V$ to $V^{\prime}$, i.e.

$$
\begin{aligned}
& \exists l \geq 0: \quad \forall x, y \in V, \quad\|B(x)-B(y)\|^{\prime} \leq l\|x-y\|, \\
& \exists \alpha>0: \quad \forall x, y \in V, \quad\langle B(x)-B(y), x-y\rangle \geq \alpha\|x-y\|^{2} .
\end{aligned}
$$

Let $f$ be a function from $[0, T] \times H$ to $V^{\prime}$, Lipschitz continuous with respect to its second argument and whose derivative maps the bounded sets of $L^{2}(0, T ; V)$ into bounded sets of $L^{2}\left(0, T ; V^{\prime}\right)$, i.e.:

$$
\exists L \geq 0: \quad \forall t \in[0, T], \quad \forall x_{1}, x_{2} \in H, \quad\left\|f\left(t, x_{1}\right)-f\left(t, x_{2}\right)\right\|^{\prime} \leq L\left|x_{1}-x_{2}\right|,
$$

and

$$
\forall R \geq 0, \quad \Phi(R)=\sup \left\{\left\|\frac{\partial f}{\partial t}(., v)\right\|_{L^{2}\left(0, T ; V^{\prime}\right)}:\|v\|_{L^{2}(0, T ; V)} \leq R\right\}<+\infty .
$$

Let $u_{0}$ belong to $D(A)$. We make the following regularity assumption:

$$
\exists z \in A\left(u_{0}\right): \quad f\left(0, u_{0}\right)-z-B\left(u_{0}\right) \in H .
$$

We will study the differential inclusion

$$
\begin{aligned}
& \dot{u}(t)+B(u(t))+A(u(t)) \ni f(t, u(t)), \text { a.e. on }] 0, T[, \\
& u(0)=u_{0},
\end{aligned}
$$

and its numerical approximation, which is defined as follows: let $N$ be a strictly positive integer, let the time-step be $h=T / N$, define $t_{p}=p h$ and let $U^{p}$ be the solution of the numerical scheme:

$$
\begin{aligned}
& \forall p \in\{0, \ldots, N-1\}, \quad \frac{U^{p+1}-U^{p}}{h}+B\left(U^{p+1}\right)+A\left(U^{p+1}\right) \ni f\left(t_{p}, U^{p}\right), \\
& U^{0}=u_{0} .
\end{aligned}
$$

This scheme possesses a unique solution: indeed, $A$ is maximal monotone and $B$ is continuous and coercive; according to Zeidler [18], if we denote by $j$ the injection $V \hookrightarrow V^{\prime}$, then, for all $\lambda>0$, the operator $(j+\lambda A+\lambda B)^{-1}$ is defined on all of $V^{\prime}$ and single-valued from $V^{\prime}$ to $V$; thus, (1.5) is equivalent to

$$
\forall p \in\{0, \ldots, N-1\}, \quad U^{p+1}=(j+h A+h B)^{-1}\left(h f\left(t_{p}, U^{p}\right)+j\left(U^{p}\right)\right) .
$$

We denote by $u_{h}$ the linear interpolation of the $U^{p}$ 's at $t_{p}$. In this paper, we estimate the order of convergence of the numerical approximation $u_{h}$ of $u$ as $h$ tends to zero.

Brezis proved in [5], the existence and the uniqueness of the solution of the differential inclusion

$$
\begin{aligned}
& \dot{u}(t)+B(u(t))+\partial \phi(u(t)) \ni g(t), \text { a.e. on }] 0, T[ \\
& u(0)=u_{0},
\end{aligned}
$$

where $B$ is a pseudo-monotone mapping from the Banach space $V$ to its dual $V^{\prime}, g$ is a function from $L^{2}\left(0, T ; V^{\prime}\right)$ and $\partial \phi$ is the sub-differential of a convex proper and lower semi-continuous function $\phi$ from $V$ to $]-\infty,+\infty]$; this sub-differential is defined by

$$
\forall(x, y) \in V \times V^{\prime}, \quad y \in \partial \phi(x) \Longleftrightarrow \forall z \in V, \quad \phi(z)-\phi(x) \geq\langle y, z-x\rangle .
$$


According to Proposition 32.17 p. 860 of [18], the sub-differential $\partial \phi$ is a maximal monotone operator from $V$ to $V^{\prime}$. The functional frame of (1.8) and (1.9) involves Banach spaces, where we use only Hilbert spaces. However, we consider the case of a right hand side which depends on the unknown $u$ and an operator $A$ which is not necessarily a sub differential.

If $V=H=V^{\prime}$ and $A$ is a maximal monotone operator whose domain has non empty interior, and non necessarily equal to a sub-differential, Brezis also proved that there exists a unique solution $u \in W^{1,1}(0, T ; H)$ of (1.3) and (1.4) (see Prop. 3.13 p. 107 of [6]).

If $V=H=V^{\prime}$, there are a few results on the convergence of $u_{h}$ to $u$ : convergence of non-linear semigroups [11], finite difference schemes for variationnal inequalities [12]. The convergence of (1.5), (1.6) has been proved by Crandall and Evans [7] and by Kartsatos [13] for an $m$-accretive operator in a Banach space. Here our functional hypotheses are stronger, which enables us to obtain an order for the convergence.

Finite dimensional results analogous to ours can be found in $[9,10,14]$ and [17]. Dontchev studied in [10] a differential inclusion without uniqueness for which he showed that there exists a discrete solution, approximating one of the many exact solutions with an order of precision larger than one; however, this result is non constructive since it does not tell us how to obtain the appropriate discrete solution. The author also proposed a scheme in order to discretize inclusions similar to (1.3) and (1.4); an order of convergence higher than one holds only on intervals where the solution is smooth enough, which is seldom true (see Rem. 1.2); without the regularity assumption, Dontchev obtained error estimates in $O\left(h^{3 / 2}\right)$ or in $O\left(h^{2}\right)$, under hypothesis that, for all $x$ in $H$, $A(x)$ is compact, which does not hold in our study.

In [16], which inspired this work, Lippold assumed (1.2a), (1.2b), $A=\partial \phi$ and

$$
\exists \lambda \geq 0, \quad \forall x, y \in \operatorname{dom} \phi, \quad|\phi(x)-\phi(y)| \leq \lambda\|x-y\|,
$$

in order to study the differential inclusion (1.8) and (1.9). Moreover, the right hand side $g$ depends only on $t$ and belongs to $H^{1}\left(0, T ; V^{\prime}\right)$; Lippold's numerical scheme is

$$
\begin{aligned}
& \forall p \in\{0, \ldots, N-1\}, \quad \frac{U^{p+1}-U^{p}}{h}+B\left(U^{p+1}\right)+\partial \phi\left(U^{p+1}\right) \ni g\left(t_{p+1}\right), \\
& U^{0}=u_{0, N},
\end{aligned}
$$

and he showed that if $\left|u_{0}-u_{0, N}\right|=O(\sqrt{h})$, then

$$
\left\|u-u_{h}\right\|_{W}=O(\sqrt{h})
$$

here, the norm $\|\cdot\|_{W}$ is defined on the Banach space $W=L^{2}(0, T ; V) \cap C^{0}([0, T], H)$ by

$$
\forall v \in W, \quad\|v\|_{W}=\max _{t \in[0, T]}\left(|v(t)|^{2}+\int_{0}^{t}\|v(s)\|^{2} \mathrm{~d} s\right)^{\frac{1}{2}} .
$$

Moreover, if

$$
g(0)-B\left(u_{0}\right) \in H
$$

holds, which is a particular case of (1.2e), and if $\left|u_{0}-u_{0, N}\right|=O(h)$ and $\phi$ is the indicatrix of a closed convex set, i.e.

$$
\phi(x)= \begin{cases}0 & \text { if } x \in K \\ +\infty & \text { if } x \notin K\end{cases}
$$


then, Lippold showed

$$
\left\|u-u_{h}\right\|_{W}=O(h) .
$$

The choice of $g\left(t_{p+1}\right)$ instead of $g\left(t_{p}\right)$ in the right hand side of (1.12) is a minor modification which does not change the order of convergence.

This paper is organized as follows: in Section 2, we give a simple proof of convergence of (1.5) and (1.6) to the solution of (1.3) and (1.4), under assumptions (1.2). For this purpose, we prove that $\left\|u_{h}-u_{k}\right\|$ is bounded by $M \sqrt{h+k}$ where $M$ does not depend on $h$ and $k$. Then, we can infer that $\left(u_{h}\right)_{h>0}$ is a Cauchy sequence and converges; moreover, the order of convergence is $1 / 2$, which generalizes Lippold's results [16]. This proof provides also estimates on $\left\|u_{h}\right\|$ and $\left\|\dot{u}_{h}\right\|$ uniformly in $h$ which we will use in what follows. In this section, we also prove the existence and the uniqueness of the solution of the differential inclusion (1.3) and (1.4). In Section 3, we obtain order of precision 1 of the scheme, if $A$ is the subdifferential of the indicatrix of a closed convex set, which generalizes Lippold's results [16].

The relative situation of our result and Lippold's is more delicate than appears naïvely: assumption (1.2e) is very strong: we show in Proposition 2.6 that it implies that $\dot{u}$ belongs to $L^{\infty}(0, T ; H)$. We may dispense with this assumption, provided that

$$
V=H=V^{\prime},
$$

then, we find all Lippold's results; Section 4 shows how to modify the proofs when assumption (1.18) holds; in this case, assumption (1.2e) is automatically verified. Assumption (1.18) is important for us, since it holds for Prandtl's rheological model which however does not involve a coercive term $B$; assumption (1.18) enables us to study the numerical scheme

$$
\begin{aligned}
& \forall p \in\{0, \ldots, N-1\}, \quad \frac{U^{p+1}-U^{p}}{h}+A\left(U^{p+1}\right) \ni f\left(t_{p}, U^{p}\right), \\
& U_{0}=u_{0},
\end{aligned}
$$

which is an approximation of the differential inclusion

$$
\begin{aligned}
& \dot{u}(t)+A(u(t)) \ni f(t, u(t)), \text { a.e. on }] 0, T[, \\
& u(0)=u_{0},
\end{aligned}
$$

where $A$ is maximal monotone and $f$ satisfies $(1.2 \mathrm{c})$ and $(1.2 \mathrm{~d})$.

The previous study was purely Hilbertian. In order to apply our results to Prandtl's model, we sought estimates in $\mathbb{R}^{n}$ equipped with the $l^{q}$ norm:

$$
\forall\left(u_{1}, \ldots, u_{n}\right) \in \mathbb{R}^{n}, \quad\left|\left(u_{1}, \ldots, u_{n}\right)\right|_{q}= \begin{cases}\left(\sum_{i=1}^{n}\left|u_{i}\right|^{q}\right)^{1 / q} & \text { if } q<+\infty \\ \max _{1 \leq i \leq n}\left|u_{i}\right| & \text { if } q=+\infty\end{cases}
$$

we shall denoted by $l_{n}^{q}$ this space. For $n=1$, it is not difficult to obtain good estimates; for an arbitrary $n$, if $K$ is a Cartesian product of non empty closed intervals

$$
K=K_{1} \times \ldots \times K_{n} .
$$

Then we can obtain $l_{n}^{q}$ estimates for the solution provided that the function $f$ satisfies the following two properties

$$
\forall t \in[0, T], \quad \forall x_{1}, x_{2} \in \mathbb{R}^{n}, \quad\left|f\left(t, x_{1}\right)-f\left(t, x_{2}\right)\right|_{q} \leq L\left|x_{1}-x_{2}\right|_{q}
$$


and for all $R \geq 0$

$$
\sup _{|x|_{q} \leq R}\left\|\frac{\partial f}{\partial t}(., v)\right\|_{L^{\infty}\left(0, T ; l_{n}^{q}\right)}<+\infty .
$$

Moreover, in Section 5, we obtain estimates

$$
\left\|u-u_{h}\right\| \leq C h^{\alpha}(\text { where } \alpha=1 / 2 \text { or } 1)
$$

with $C$ independent of $h$. In Section 6 , we give the exact form of the dependence of $C$ in terms of $n, q, T, L$, $f\left(., u_{0}\right)$ and $\partial f / \partial t$; this enables us to show in Section 7 that, in the case of Prandtl's model, the estimates in maximum norm are uniform with respect to $n$.

In Section 8, we will present simulations which suggested us the order of convergence proved here: they show the observation on the order which motivated this work.

Remark 1.1. The order of scheme (1.5) and (1.6) cannot be greater than one. If $A=0, B=\mathbf{1}, f(t, u)=$ $g(t, u)+u$, then we have an ordinary differential equations and (1.3) and (1.4) is nothing but the explicit Euler method known to be of order one.

Remark 1.2. The solution of (1.3) and (1.4) is not necessarily of class $C^{1}$, even for a very smooth function $f$. We conjecture that none of the classical schemes of order 2 can give an approximation of order 1 for this type of problem; let us check that for Crank-Nicolson's scheme, assuming

$$
T=2, \quad B(u)=u, \quad A=\partial \psi_{\mathbb{R}_{+}}, \quad u_{0}=-1 \text { and } f(t, u)=u-1
$$

The solution of (1.3) and (1.4) is given then by

$$
\forall t \in[0,2], \quad u(t)=(1-t)^{+}
$$

and the scheme

$$
\begin{aligned}
& \forall p \in\{0, \ldots, N-1\}, \quad \frac{U^{p+1}-U^{p}}{h}+\partial \psi_{\mathbb{R}_{+}}\left(\frac{U^{p+1}+U^{p}}{2}\right) \ni-1, \\
& U^{0}=u_{0}
\end{aligned}
$$

has a solution given by

$$
\forall q \in\{0, \ldots, N\}, \quad U^{p}= \begin{cases}1-p h & \text { if } p \leq m \\ (-1)^{p-m}(1-m h) & \text { if } p>m\end{cases}
$$

where $m$ is the largest integer which is strictly less than $1 / h+1 / 2$ (see Fig. 1 ). Then we may write

$$
\left.\left.\frac{1}{h}=m+\xi, \quad \xi \in\right]-1 / 2,1 / 2\right]
$$

and as $h$ tends to zero, $\xi$ goes through all the values in $]-1 / 2,1 / 2]$; it is plain that

$$
\left|\frac{1-m h}{h}\right|=|\xi|
$$

and therefore the scheme is at most of order one. 


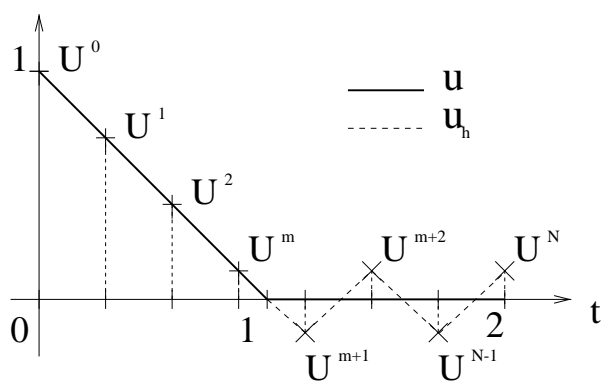

Figure 1. Exact and approximated solutions of system (1.3) and (1.4) under assumptions (1.25).

\section{Convergence, existence, uniqueness And order $1 / 2$}

We first prove the uniqueness of the solution of (1.3) and (1.4).

Proposition 2.1. Let $A$ be a maximal monotone operator from $V$ to $V^{\prime}, u_{0} \in D(A)$, with $f$ and $B$ verifying $(1.2 \mathrm{~b})$ and $(1.2 \mathrm{c})$. There exists at most one function u belonging to $L^{2}(0, T ; V)$ whose derivative belongs to $L^{2}\left(0, T ; V^{\prime}\right)$ and verifying (1.3) and (1.4).

Proof. This proof is based on Gronwall's lemma. Let $u_{1}$ and $u_{2}$ be two solutions of (1.3) and (1.4), belonging to $L^{2}(0, T ; V)$ whose derivatives belongs to $L^{2}\left(0, T ; V^{\prime}\right)$. We recall that for all function $u$ belonging to $L^{2}(0, T ; V)$ whose derivative belongs to $L^{2}\left(0, T ; V^{\prime}\right)$ and for all $v$ in $V$

$$
\langle\dot{u}(.), v\rangle=\frac{\mathrm{d}}{\mathrm{d} t}(u(.), v), \text { in } \mathcal{D}^{\prime}(] 0, T[) ;
$$

see for example [8]. We multiply the difference between (1.3) applied to $u_{1}$ and (1.3) applied to $u_{2}$ by $u_{1}-u_{2}$, we use the monotonicity, we integrate in time and we find

$$
\begin{aligned}
\forall t \in[0, T], \quad \frac{1}{2}\left|u_{1}(t)-u_{2}(t)\right|^{2}+\int_{0}^{t}\left\langle B\left(u_{1}(s)\right)-B\left(u_{2}(s)\right), u_{1}(s)-u_{2}(s)\right\rangle \mathrm{d} s & \\
\leq & \leq \int_{0}^{t}\left\langle f\left(s, u_{1}(s)\right)-f\left(s, u_{2}(s)\right), u_{1}(s)-u_{2}(s)\right\rangle \mathrm{d} s
\end{aligned}
$$

According to (1.2b) and (1.2c), we have

$$
\forall t \in[0, T], \quad \frac{1}{2}\left|u_{1}(t)-u_{2}(t)\right|^{2}+\alpha \int_{0}^{t}\left\|u_{1}(s)-u_{2}(s)\right\|^{2} \mathrm{~d} s \leq L \int_{0}^{t}\left|u_{1}(s)-u_{2}(s)\right|\left\|u_{1}(s)-u_{2}(s)\right\| \mathrm{d} s .
$$

Choose $\varepsilon \leq \alpha / L$ in the classical inequality

$$
\forall \varepsilon>0, \quad \forall a, b \in \mathbb{R}, \quad a b \leq \frac{1}{2}\left(\varepsilon a^{2}+\frac{b^{2}}{\varepsilon}\right)
$$


we then obtain

$$
\forall t \in[0, T], \quad \frac{1}{2}\left|u_{1}(t)-u_{2}(t)\right|^{2}+\frac{\alpha}{2} \int_{0}^{t}\left\|u_{1}(s)-u_{2}(s)\right\|^{2} \mathrm{~d} s \leq \frac{L^{2}}{2 \alpha} \int_{0}^{t}\left|u_{1}(s)-u_{2}(s)\right|^{2} \mathrm{~d} s .
$$

It is immediate now that $u_{1}=u_{2}$.

Let us establish now estimates on $u_{h}$ and $\dot{u}_{h}$ independently of $h$ :

Lemma 2.2. We assume that (1.2b), (1.2c), (1.2d) and (1.2e) hold. There exists a constant $M$ depending only on $T, L, \alpha, \Phi$ and $u_{0}$ such that for all $N \in \mathbb{N}^{*}$,

$$
\begin{aligned}
& \left\|u_{h}-u_{0}\right\|_{C^{0}([0, T], H)} \leq M, \\
& \left\|u_{h}-u_{0}\right\|_{L^{2}(0, T ; V)} \leq M \\
& \left\|\dot{u}_{h}\right\|_{L^{\infty}(0, T ; H)} \leq M \\
& \left\|\dot{u}_{h}\right\|_{L^{2}(0, T ; V)} \leq M
\end{aligned}
$$

Proof. For the discrete solution, a discrete Gronwall's lemma enables us to estimate $U^{p}-u_{0}$ and $\left(U^{p+1}-U^{p}\right) / h$.

Let $z \in A\left(u_{0}\right)$ and

$$
w=z+B\left(u_{0}\right)-f\left(0, u_{0}\right)
$$

The numerical scheme (1.5) can be rewritten under the form

$$
\forall p \in\{0, \ldots, N-1\}, \quad U^{p+1}+h\left(B\left(U^{p+1}\right)+A\left(U^{p+1}\right)\right) \ni h f\left(t_{p}, U^{p}\right)+U^{p} .
$$

We multiply the difference between $(2.7)$ and (2.8) by $U^{p+1}-u_{0}$ and we obtain

$$
\begin{aligned}
\forall p \in\{0, \ldots, N-1\}, \quad\left\langle U^{p+1}-u_{0}, U^{p+1}-u_{0}\right\rangle+h\left\langle B\left(U^{p+1}\right)-B\left(u_{0}\right), U^{p+1}-u_{0}\right\rangle \leq \\
h\left\langle f\left(t_{p}, U^{p}\right)-f\left(0, u_{0}\right)-w, U^{p+1}-u_{0}\right\rangle+\left\langle U^{p}-u_{0}, U^{p+1}-u_{0}\right\rangle .
\end{aligned}
$$

Thanks to the classical identification

$$
\forall x \in H, \quad \forall y \in V, \quad\langle x, y\rangle=(x, y),
$$

hypotheses (1.2b) and (1.2c), and the triangular inequality, we get

$$
\begin{aligned}
\left|U^{p+1}-u_{0}\right|^{2}+h \alpha\left\|U^{p+1}-u_{0}\right\|^{2} \leq & L h\left|U^{p}-u_{0}\right|\left\|U^{p+1}-u_{0}\right\| \\
& +h\left\|f\left(t_{p}, u_{0}\right)-f\left(0, u_{0}\right)-w\right\|^{\prime}\left\|U^{p+1}-u_{0}\right\|+\left|U^{p+1}-u_{0}\right|\left|U^{p}-u_{0}\right|
\end{aligned}
$$

Each of the three products on the right hand side of (2.10) is smaller than a linear combination of squares as in (2.2); in each of these combinations we have to choose the parameter $\varepsilon$; after completing the calculations, we obtain:

$\left|U^{p+1}-u_{0}\right|^{2}+h \alpha\left\|U^{p+1}-u_{0}\right\|^{2} \leq\left|U^{p}-u_{0}\right|^{2}+\frac{2 L^{2} h}{\alpha}\left|U^{p}-u_{0}\right|^{2}+\frac{2 h}{\alpha}\left(\left\|f\left(t_{p}, u_{0}\right)-f\left(0, u_{0}\right)-w\right\|^{\prime}\right)^{2}$

According to the discrete Gronwall's lemma, we obtain

$$
\forall p \in\{0, \ldots, N\}, \quad\left|U^{p}-u_{0}\right|^{2}+h \alpha\left\|U^{p}-u_{0}\right\|^{2} \leq \frac{2}{\alpha} \mathrm{e}^{2 L^{2} T / \alpha} \sum_{p=0}^{N} h\left\|f\left(t_{p}, u_{0}\right)-f\left(0, u_{0}\right)-w\right\|^{\prime 2} .
$$


According to assumption $(1.2 \mathrm{~d})$, the term $\sum_{p=0}^{N} h\left(\left\|f\left(t_{p}, u_{0}\right)-f\left(0, u_{0}\right)-w\right\|^{\prime}\right)^{2}$ is bounded uniformly in $N$; thus, $\left|U^{p}-u_{0}\right|^{2}+h \alpha\left\|U^{p}-u_{0}\right\|^{2}$ is bounded uniformly in $N$ and in $p$, which gives (2.3). By summing the estimates (2.11) for $p \in\{0, \ldots, N-1\}$, we can deduce (2.4).

Let us prove now (2.5) and (2.6). Denoting by $V^{p}$ the discrete speed defined by

$$
\forall p \in\{0, \ldots, N-1\}, \quad V^{p}=\frac{U^{p+1}-U^{p}}{h},
$$

we rewrite the numerical scheme (1.5) under the form

$$
\forall p \in\{0, \ldots, N-1\}, \quad V^{p}+A\left(U^{p+1}\right)+B\left(U^{p+1}\right) \ni f\left(t_{p}, U^{p}\right) .
$$

We multiply the difference of (2.13) for $p$ with (2.13) for $p-1$ by $V^{p}$ :

$$
\begin{aligned}
& \forall p \in\{1, \ldots, N-1\}, \quad\left\langle V^{p}-V^{p-1}, V^{p}\right\rangle+\frac{1}{h}\left\langle B\left(U^{p+1}\right)-B\left(U^{p}\right), U^{p+1}-U^{p}\right\rangle \leq \\
& \left\langle f\left(t_{p}, U^{p}\right)-f\left(t_{p-1}, U^{p-1}\right), V^{p}\right\rangle .
\end{aligned}
$$

As in (2.11), we obtain easily

$$
\forall p \in\{1, \ldots, N-1\}, \quad\left|V^{p}\right|^{2}+h \alpha\left\|V^{p}\right\|^{2} \leq\left|V^{p-1}\right|^{2}+\frac{2 L^{2} h}{\alpha}\left|V^{p-1}\right|^{2}+\frac{2}{\alpha h}\left\|\delta_{p}\right\|^{\prime 2},
$$

where

$$
\delta_{p}=f\left(t_{p}, U^{p-1}\right)-f\left(t_{p-1}, U^{p-1}\right) .
$$

The discrete Gronwall's lemma implies:

$$
\forall p \in\{0, \ldots, N\}, \quad\left|V^{p}\right|^{2}+h \alpha\left\|V^{p}\right\|^{2} \leq \mathrm{e}^{2 L^{2} T / \alpha}\left(\left|V^{0}\right|^{2}+h \alpha\left\|V^{0}\right\|^{2}+\frac{2}{\alpha h} \sum_{p=1}^{N-1}\left\|\delta_{p}\right\|^{2}\right) .
$$

We denote by $\widetilde{v}_{h}$ the function of $L^{2}(0, T ; V)$ defined by

$$
\forall p \in\{0, \ldots, N-1\}, \quad \forall t \in] t_{p}, t_{p+1}\left[, \quad \widetilde{v}_{h}(t)=U^{p} ;\right.
$$

according to the Cauchy-Schwarz's inequality, we obtain

$$
\frac{1}{h} \sum_{p=1}^{N-1}\left\|\delta_{p}\right\|^{2} \leq \int_{0}^{T}\left\|\frac{\partial f}{\partial t}\left(s, \widetilde{v}_{h}(s)\right)\right\|^{2} \mathrm{~d} s
$$

and thus, for all $p \in\{0, \ldots, N\}$

$$
\left|V^{p}\right|^{2}+h \alpha\left\|V^{p}\right\|^{2} \leq \mathrm{e}^{2 L^{2} T / \alpha}\left(\left|V^{0}\right|^{2}+h \alpha\left\|V^{0}\right\|^{2}+\frac{2}{\alpha} \int_{0}^{T}\left(\left\|\frac{\partial f}{\partial t}\left(s, \widetilde{v}_{h}(s)\right)\right\|^{\prime}\right)^{2} \mathrm{~d} s\right) .
$$

Therefore, assumption (1.2e) implies that the initial discrete speed $V^{0}$ is bounded uniformly in $N$ : indeed, (1.2e) can be rewritten as

$$
\exists w \in H, \quad w+B\left(u_{0}\right)+A\left(u_{0}\right) \ni f\left(0, u_{0}\right) ;
$$


if we subtract this relation from (2.13) for $p=0$, we obtain according to (1.2b),

$$
\left|V^{0}\right|^{2}+\alpha h\left\|V^{0}\right\|^{2} \leq-\left\langle w, V^{0}\right\rangle,
$$

which implies immediately

$$
\frac{1}{2}\left|V^{0}\right|^{2}+\alpha h\left\|V^{0}\right\|^{2} \leq \frac{1}{2}|w|^{2} .
$$

Finally, estimate (2.4) implies that the function $\widetilde{v}_{h}$ belongs to a bounded subset of $L^{2}(0, T ; V)$; then, thanks to assumptions (1.2d) and relations (2.18) and (2.19), there exists a $C$ which depends on $T, L, \alpha, \Phi$ and $u_{0}$ such that

$$
\forall p \in\{0, \ldots, N\}, \quad\left|V^{p}\right|^{2}+h \alpha\left\|V^{p}\right\|^{2} \leq C .
$$

This estimate implies (2.5). By summing (2.15), we obtain (2.6).

Let us prove now the convergence of the numerical scheme, which proves also the existence of the solution of (1.3) and (1.4).

We might think that the estimates obtained at Lemma 2.2 are sufficient for passing to the limit; define piecewise constant functions: for all $p \in\{0, \ldots, N-1\}$

$$
\begin{aligned}
v_{h}(t) & =U^{p+1} \text { on }\left[t_{p}, t_{p+1}[,\right. \\
\widetilde{v}_{h}(t) & =U^{p} \text { on }\left[t_{p}, t_{p+1}[,\right. \\
f_{h}(t) & =f\left(t_{p}, U^{p}\right) \text { on }\left[t_{p}, t_{p+1}[\right.
\end{aligned}
$$

and let $u_{h}$ be the piecewise linear interpolation taking the value $U^{p}$ at $t_{p}$. Then $u_{h}$ satisfies the equation

$$
\dot{u}_{h}+A\left(v_{h}\right) \ni f_{h}-B\left(v_{h}\right) \text {, a.e. on }[0, T] .
$$

The classical method for passing to the limit requires at least the following information:

$$
\limsup _{h \rightarrow 0_{+}} \int_{0}^{T}\left\langle f_{h}-B\left(v_{h}\right)-\dot{u}_{h}, u_{h}\right\rangle \mathrm{d} t \leq \int_{0}^{T}\langle f-B(v)-\dot{u}, u\rangle \mathrm{d} t .
$$

The term containing the time derivative can be handled by integration; the other terms cannot be handled unless we know something about the strong convergence of $u_{h}$ in $L^{2}(0, T ; H)$; this would be true if the injection $V \hookrightarrow H$ were compact. But we did not make this assumption and we do not need it.

We stated that the convergence is of order $1 / 2$; this means that we have metric result, which is much stronger than a topological result. In particular, we are going to prove that the sequence $\left(u_{h}\right)_{h>0}$ is a Cauchy sequence and we shall estimate $\left\|u_{h}-u_{k}\right\|_{L^{\infty}(0, T ; H)}$ and $\left\|u_{h}-u_{k}\right\|_{L^{2}(0, T ; V)}$ in term of $\sqrt{h+k}$. These estimates depend on a couple of preliminary lemmas which strongly use the regularity assumptions.

Lemma 2.3. If assumptions (1.2b), (1.2c), (1.2d) and (1.2e) hold, then there exists a constant $M_{1}$ depending only on $T, L, \alpha, \Phi$ and $u_{0}$ such that for all $h>0$

$$
\left\|u_{h}-v_{h}\right\|_{L^{2}(0, T ; H)}+\left\|u_{h}-v_{h}\right\|_{L^{2}(0, T ; V)}+\left\|u_{h}-\widetilde{v}_{h}\right\|_{L^{2}(0, T ; H)}+\left\|u_{h}-\widetilde{v}_{h}\right\|_{L^{2}(0, T ; V)} \leq M_{1} h .
$$

Proof. By definition of $u_{h}$ and $v_{h}$, on the interval $\left[t_{p}, t_{p+1}[\right.$

$$
u_{h}(t)-v_{h}(t)=U^{p}+\frac{t-t_{p}}{h}\left(U^{p+1}-U^{p}\right)-U^{p+1}=\left(U^{p+1}-U^{p}\right)\left(-1+\frac{t-t_{p}}{h}\right) .
$$


Therefore, by integration over $\left[t_{p}, t_{p+1}[\right.$,

$$
\int_{t_{p}}^{t_{p+1}}\left|u_{h}(t)-v_{h}(t)\right|^{2} \mathrm{~d} t=\left|\frac{U^{p+1}-U^{p}}{h}\right|^{2} \frac{h^{3}}{3}
$$

and similarly

$$
\int_{t_{p}}^{t_{p+1}}\left\|u_{h}(t)-v_{h}(t)\right\|^{2} \mathrm{~d} t=\left\|\frac{U^{p+1}-U^{p}}{h}\right\|^{2} \frac{h^{3}}{3}
$$

Thanks to $(2.5)$, we see that

$$
\int_{0}^{T}\left|u_{h}(t)-v_{h}(t)\right|^{2} \mathrm{~d} t=\frac{h^{3}}{3} \sum_{p=0}^{N-1}\left|\frac{U^{p+1}-U^{p}}{h}\right|^{2} \leq \frac{h^{3}}{3} N\left\|\dot{u}_{h}\right\|_{L^{\infty}(0, T ; H)} \leq \frac{h^{2} T M}{3} .
$$

Similarly, (2.6) implies

$$
\int_{0}^{T}\left\|u_{h}(t)-v_{h}(t)\right\|^{2} \mathrm{~d} t=\frac{h^{2}}{3} \sum_{p=0}^{N-1}\left\|\frac{U^{p+1}-U^{p}}{h}\right\|^{2} h=\frac{h^{2}}{3}\left\|\dot{u}_{h}\right\|_{L^{2}(0, T ; V)}^{2} \leq \frac{h^{2} M^{2}}{3} .
$$

The estimates pertaining to $u_{h}-\widetilde{v}_{h}$ are proved in an analogous and left to the reader.

Lemma 2.4. If assumptions (1.2b), (1.2c), (1.2d) and (1.2e) hold, then there exists a constant $M_{2}$ depending only on $T, L, \alpha, \Phi$ and $u_{0}$ such that for all $h>0$ :

$$
\left\|f\left(., \widetilde{v}_{h}\right)-f_{h}\right\|_{L^{2}\left(0, T ; V^{\prime}\right)} \leq M_{2} h .
$$

Proof. The function $f_{h}$ is constant and equal to $f\left(t_{p}, U^{p}\right)$ over $\left[t_{p}, t_{p+1}[\right.$; therefore, on that interval,

$$
f\left(t, \widetilde{v}_{h}(t)\right)-f_{h}(t)=\int_{t_{p}}^{t} \frac{\partial f}{\partial t}\left(s, U^{p}\right) \mathrm{d} s .
$$

We apply Cauchy-Schwarz inequality

$$
\left(\left\|f\left(t, \widetilde{v}_{h}(t)\right)-f_{h}(t)\right\|^{\prime}\right)^{2} \leq\left(t-t_{p}\right) \int_{t_{p}}^{t}\left(\left\|\frac{\partial f}{\partial t}\left(s, U^{p}\right)\right\|^{\prime}\right)^{2} \mathrm{~d} s .
$$

We integrate this inequality over $\left[t_{p}, t_{p+1}[\right.$, obtaining

$$
\int_{t_{p}}^{t_{p+1}}\left(\left\|f\left(t, \widetilde{v}_{h}(t)\right)-f_{h}(t)\right\|^{\prime}\right)^{2} \mathrm{~d} t \leq \frac{h^{2}}{2} \int_{t_{p}}^{t_{p+1}}\left(\left\|\frac{\partial f}{\partial t}\left(s, U^{p}\right)\right\|^{\prime}\right)^{2} \mathrm{~d} s .
$$

If we sum these inequalities with respect to $p$, we get

$$
\int_{0}^{T}\left(\left\|f\left(t, \widetilde{v}_{h}(t)\right)-f_{h}(t)\right\|^{\prime}\right)^{2} \mathrm{~d} t \leq \frac{h^{2}}{2} \int_{0}^{T}\left(\left\|\frac{\partial f}{\partial t}\left(s, \widetilde{v}_{p}(s)\right)\right\|^{\prime}\right)^{2} \mathrm{~d} s
$$


otherwise, we have

$$
\left\|\widetilde{v}_{h}\right\|_{L^{2}(0, T ; V)} \leq\left\|\widetilde{v}_{h}-u_{h}\right\|_{L^{2}(0, T ; V)}+\left\|u_{h}-u_{0}\right\|_{L^{2}(0, T ; V)}+\left\|u_{0}\right\|_{L^{2}(0, T ; V)} \leq M_{1} T+M+\sqrt{T}\left\|u_{0}\right\| .
$$

Then, we have, according to $(1.2 \mathrm{~d})$,

$$
\int_{0}^{T}\left(\left\|f\left(t, \widetilde{v}_{h}(t)\right)-f_{h}(t)\right\|^{\prime}\right)^{2} \mathrm{~d} t \leq \frac{h^{2}}{2}\left(\Phi\left(M_{1} T+M+\sqrt{T}\left\|u_{0}\right\|\right)\right)^{2},
$$

which concludes the proof of the lemma.

We are now able to estimate the difference $u_{h}-u_{k}$ for all $h, k$.

Proposition 2.5. If assumptions (1.2b), (1.2c), (1.2d) and (1.2e) hold, then there exists a constant $M_{3}$ depending only on $T, L, \alpha, \Phi$ and $u_{0}$ such that for all $h, k$ and all $t$ in $[0, T]$ :

$$
\left|u_{h}(t)-u_{k}(t)\right|^{2}+\int_{0}^{t}\left\|u_{h}(s)-u_{k}(s)\right\|^{2} \mathrm{~d} s \leq M_{3}(h+k) .
$$

Proof. The equations satisfied respectively by $u_{h}$ and $u_{k}$ are

$$
\begin{gathered}
\dot{u}_{h}+A v_{h}+B v_{h} \ni f_{h}, \\
\dot{u}_{k}+A v_{k}+B v_{k} \ni f_{k} .
\end{gathered}
$$

If we subtract (2.26) from (2.25), allowing for the usual abuse of notations, and multiply by $v_{h}-v_{k}$, we obtain the inequality

$$
\left(\dot{u}_{h}-\dot{u}_{k}, v_{h}-v_{k}\right)+\alpha\left\|v_{h}-v_{k}\right\|^{2} \leq\left\langle f_{h}-f_{k}, v_{h}-v_{k}\right\rangle \cdot
$$

The first term in the left hand side of $(2.27)$ is integrated over $[0, t]$ and rewritten as

$$
\frac{1}{2}\left|u_{h}(t)-u_{k}(t)\right|^{2}+\int_{0}^{t}\left(\dot{u}_{h}-\dot{u}_{k}, v_{h}-u_{h}-v_{k}+u_{k}\right) \mathrm{d} s .
$$

But we can estimate the absolute value of the above integral by Cauchy-Schwarz inequality

$$
\begin{aligned}
& \left|\int_{0}^{t}\left(\dot{u}_{h}-\dot{u}_{k}, v_{h}-u_{h}-v_{k}+u_{k}\right) \mathrm{d} s\right| \\
\leq & \left(\left\|\dot{u}_{h}\right\|_{L^{2}(0, T: H)}+\left\|\dot{u}_{k}\right\|_{L^{2}(0, T: H)}\right)\left(\left\|v_{h}-u_{h}\right\|_{L^{2}(0, T: H)}+\left\|v_{k}-u_{k}\right\|_{L^{2}(0, T: H)}\right) \\
\leq & 2 M M_{1}(h+k) .
\end{aligned}
$$

In order to estimate the integral over $[0, t]$ of the right hand side of $(2.27)$, we apply the triangle inequality and $(1.2 \mathrm{c})$

$$
\left\|f_{h}-f_{k}\right\|^{\prime} \leq\left\|f_{h}-f\left(., \widetilde{v}_{h}\right)\right\|^{\prime}+\left\|f_{k}-f\left(., \widetilde{v}_{k}\right)\right\|^{\prime}+L\left|u_{h}-\widetilde{v}_{h}\right|+L\left|u_{k}-\widetilde{v}_{k}\right|+L\left|u_{h}-u_{k}\right| .
$$

Therefore, by Cauchy-Schwarz inequality,

$$
\begin{aligned}
\int_{0}^{t}\left\langle f_{h}-f_{k}, v_{h}-v_{k}\right\rangle \mathrm{d} s \leq 2\left(\left(M_{2}(h+k)+L M_{1}(h+k)\right)\left(\int_{0}^{t}\left\|v_{h}-v_{k}\right\|^{2} \mathrm{~d} s\right)^{\frac{1}{2}}\right. & +L \int_{0}^{t}\left|u_{h}-u_{k}\right|\left\|v_{h}-v_{k}\right\| \mathrm{d} s .
\end{aligned}
$$


We apply once again the classical inequality (2.2) with parameter $\varepsilon_{1}$ in the first product of the right hand side of $(2.28)$ and $\varepsilon_{2}$ in the second one; then

$$
\begin{aligned}
\frac{1}{2} \mid u_{h}(t) & -\left.u_{k}(t)\right|^{2}+\alpha \int_{0}^{t}\left\|v_{h}(s)-v_{k}(s)\right\|^{2} \mathrm{~d} s \leq \frac{1}{\varepsilon_{1}}\left(\left(M_{2}+L M_{1}\right)(h+k)\right)^{2} \\
& +\varepsilon_{1} \int_{0}^{t}\left\|v_{h}(s)-v_{k}(s)\right\|^{2} \mathrm{~d} s+\frac{L}{2 \varepsilon_{2}} \int_{0}^{t}\left|u_{h}(s)-u_{k}(s)\right|^{2} \mathrm{~d} s+\frac{L \varepsilon_{2}}{2} \int_{0}^{t}\left\|v_{h}(s)-v_{k}(s)\right\|^{2} \mathrm{~d} s+2 M M_{1}(h+k) .
\end{aligned}
$$

If we chose $\varepsilon_{1}$ and $\varepsilon_{2}$ such that $\varepsilon_{1}+L \varepsilon_{2} / 2<\alpha$, we obtain the following Gronwall's inequality

$$
\begin{aligned}
& \frac{1}{2}\left|u_{h}(t)-u_{k}(t)\right|^{2}+\left(\alpha-\left(\varepsilon_{1}+\frac{L \varepsilon_{2}}{2}\right)\right) \int_{0}^{t}\left\|v_{h}(s)-v_{k}(s)\right\|^{2} \mathrm{~d} s \\
& \quad \leq(h+k)\left(2 M M_{1}+\frac{\left(M_{2}+L M_{1}\right)^{2} T}{\varepsilon_{1}}\right)+\frac{L}{2 \varepsilon_{2}} \int_{0}^{t}\left|u_{h}(s)-u_{k}(s)\right|^{2} \mathrm{~d} s .
\end{aligned}
$$

This implies immediately the estimate

$$
\frac{1}{2}\left|u_{h}(t)-u_{k}(t)\right|^{2}+\left(\alpha-\left(\varepsilon_{1}+\frac{L \varepsilon_{2}}{2}\right)\right) \int_{0}^{t}\left\|v_{h}(s)-v_{k}(s)\right\|^{2} \mathrm{~d} s \leq M_{3}^{\prime}(h+k),
$$

where $M_{3}^{\prime}$ depends only on $L, T, \alpha, M, \Phi$ and $u_{0}$. If we wish the estimate $\int_{0}^{t}\left\|u_{h}(s)-u_{k}(s)\right\|^{2} \mathrm{~d} s$, we observe that we already know estimate on $\left\|u_{h}-v_{h}\right\|_{L^{2}(0, T ; V)},\left\|v_{h}-v_{k}\right\|_{L^{2}(0, T ; V)}$ and $\left\|v_{k}-u_{k}\right\|_{L^{2}(0, T ; V)}$ and the proposition is proved.

Proposition 2.6. Assume that (1.2) holds. There exists a unique solution $u$ of (1.3) and (1.4) in $C^{0}([0, T], V)$ such that $\dot{u}$ belongs to $L^{\infty}(0, T ; H)$. Moreover, if we denote by $u_{h}$ the approximation defined by (1.5) and (1.6), we have

$$
\lim _{h \rightarrow 0^{+}} \max _{t \in[0, T]}\left|u(t)-u_{h}(t)\right|^{2}+\int_{0}^{t}\left\|u(s)-u_{h}(s)\right\|^{2} \mathrm{~d} s=0 .
$$

Proof. The uniqueness of the solution is already proved. Thanks to (2.3) trough (2.6), we extract a subsequence still denoted by $\left(u_{h}\right)_{h>0}$ which converges in the following sense to a certain function $u$ :

$$
\begin{aligned}
& u_{h} \rightarrow u \text { in } L^{\infty}(0, T ; H) \text { weak } *, \\
& u_{h} \rightarrow u \text { in } L^{2}(0, T ; V) \text { weak, } \\
& \dot{u}_{h} \rightarrow \dot{u} \text { in } L^{\infty}(0, T ; H) \text { weak } *, \\
& \dot{u}_{h} \rightarrow \dot{u} \text { in } L^{2}(0, T ; V) \text { weak }
\end{aligned}
$$

Otherwise, the theory of maximal monotone operators from a Hilbert space $V$ to its dual $V^{\prime}$ is isomorphic to the standard theory of maximal monotone operators in a Hilbert space $H[6]$; let indeed $\mathcal{I}$ the duality map from $V$ to $V^{\prime}$ defined by

$$
\langle\mathcal{I} u, v\rangle=((u, v)), \quad \forall u, v \in V
$$

the interpolated space $\left[V, V^{\prime}\right]_{1 / 2}$ coincides with $H[15]$; it is a classical that the operator $\mathcal{I}_{1}$ which coincides with $\mathcal{I}$ on $D\left(\mathcal{I}_{1}\right)=\mathcal{I}^{-1} H$ is a self adjoint and that $\mathcal{I}^{1 / 2}$ is a isometry from $V$ to $H$ and its extension is also an isometry from $H$ to $V^{\prime}$. Then $A$ is maximal monotone from $V$ to $V^{\prime}$ if and only if $\mathcal{I}_{1}^{-1 / 2} A \mathcal{I}_{1}^{-1 / 2}$ is maximal 
monotone in $H$. Then, Proposition 2.2 p. 23 of [6] can be translated into the following criterion: $A$ is maximal monotone from $V$ to $V^{\prime}$ if and only if the image of $j+A$ is equal to $V^{\prime}$ where $j$ is the injection $V \hookrightarrow V^{\prime}$; the proof of this assertion is left to the reader. Similarly, if $A$ is maximal monotone from $V$ to $V^{\prime}$, the operator $\mathcal{A}$ defined by

$$
D(\mathcal{A})=\left\{u \in L^{2}(0, T ; V): \quad u(t) \in D(A), \text { a.e }\right\}
$$

and

$$
g \in \mathcal{A}(u) \Longleftrightarrow \text { a.e } g(t) \in A(u(t))
$$

is maximal monotone from $L^{2}(0, T ; V)$ to $L^{2}\left(0, T ; V^{\prime}\right)$; see example 2.3 .3 p. 25 of [6] for details. Finally, the weak convergence argument of Proposition 2.5 p. 27 of [6] is also readily translated.

According to Proposition 2.5, $\left(u_{h}\right)_{h>0}$ is a Cauchy sequence in the Banach space $W=L^{2}(0, T ; V) \cap C^{0}([0, T], H)$ and there exists $v \in W$ such that

$$
\lim _{h \rightarrow 0} u_{h}=v \text { in } W
$$

for the norm $\|.\|_{W}$ defined by (1.15). According to (2.30) trough (2.33), we have $v=u$. Define step functions $v_{h} \in L^{2}(0, T ; V)$ and $f_{h} \in L^{2}\left(0, T ; V^{\prime}\right)$ by $(2.20)$ and $(2.22)$. It is plain that $v_{h}$ converges strongly to $u$ in $L^{2}(0, T ; V)$ and $f_{h}$ converges strongly to $f(., u)$ in $L^{2}\left(0, T ; V^{\prime}\right) ; \dot{u}_{h}$ converges weakly to $\dot{u}$ in $L^{2}\left(0, T ; V^{\prime}\right)$ and $B\left(v_{h}\right)$ converges strongly to $B(u)$ in $L^{2}\left(0, T ; V^{\prime}\right)$. We infer from

$$
f_{h}-\dot{u}_{h}-B\left(u_{h}\right) \in \mathcal{A}\left(u_{h}\right)
$$

and the strong convergence of $u_{h}$ to $u$ in $L^{2}(0, T ; V)$ that in the limit

$$
f(., u)-\dot{u}-B(u) \in \mathcal{A}(u) .
$$

According to the initial condition (1.6), we have

$$
u(0)=u_{0}
$$

Thus, $u$ is solution of (1.3) and (1.4).

We obtain easily the order $1 / 2$ of convergence:

Proposition 2.7. If assumptions (1.2) hold, then scheme is of order $1 / 2$, i.e.: there exists a constant $C$ such that

$$
\forall h>0, \quad \max _{t \in[0, T]}\left(\left|u(t)-u_{h}(t)\right|^{2}+\int_{0}^{t}\left\|u(s)-u_{h}(s)\right\|^{2} \mathrm{~d} s\right)^{\frac{1}{2}} \leq C \sqrt{h}
$$

Proof. Indeed, thanks to estimate (2.24) for $k \rightarrow 0$ and (2.29), we obtain

$$
\max _{t \in[0, T]}\left|u(t)-u_{h}(t)\right|^{2}+\int_{0}^{t}\left\|u(s)-u_{h}(s)\right\|^{2} \mathrm{~d} s \leq M_{3} h
$$




\section{The SCHEME IS OF ORDER ONE IF $K$ IS A NON EMPTy CLOSED CONVEX SUBSET OF $V$ AND IF $A=\partial \psi_{K}$}

In this section, we assume that $K$ is a non empty closed convex subset of $V$ and that $A$ is the subdifferential of the indicatrix of the convex $K$ defined by (1.16). In this particular case, definition (1.10) is equivalent to:

$$
\forall(x, y) \in K \times V^{\prime}, \quad y \in \partial \phi(x) \Longleftrightarrow \forall z \in K, \quad\langle y, x-z\rangle \geq 0,
$$

and

$$
\forall x \notin K, \quad \partial \psi_{K}(x)=\emptyset .
$$

The following characteristic property of $\partial \psi_{K}$ holds:

$$
\forall x \in K, \quad 0 \in \partial \psi_{K}(x) .
$$

Proposition 3.1. Let $K$ be a non empty closed convex subset of $V$ and $A$ the maximal monotone operator $\partial \psi_{K}$. If hypotheses (1.2) hold, then the order of the scheme is one, i.e.: there exists $C$ such that

$$
\forall h>0, \quad \max _{t \in[0, T]}\left(\left|u(t)-u_{h}(t)\right|^{2}+\int_{0}^{t}\left\|u(s)-u_{h}(s)\right\|^{2} \mathrm{~d} s\right)^{\frac{1}{2}} \leq C h .
$$

Proof. By using the characteristic property (3.3), we will make difference between numerical scheme and differential inclusion and we conclude by using a Gronwall's lemma.

By construction, $u_{h}(s)$ belongs to $K$; thus, according to (3.3), we have

$$
\partial \psi_{K}\left(u_{h}(t)\right) \ni 0 \text {, a.e. on }[0, T] \text {. }
$$

If we multiply the difference between (3.5) and the differential inclusion (1.3) by $u(s)-u_{h}(s)$, we obtain

$$
\left\langle\dot{u}(s), u(s)-u_{h}(s)\right\rangle+\left\langle B(u(s)), u(s)-u_{h}(s)\right\rangle \leq\left\langle f(s, u(s)), u(s)-u_{h}(s)\right\rangle \text {, a.e. on }[0, T] .
$$

In the same way, $u(s)$ belongs to $K$ and by using the numerical scheme $(2.23)$, we obtain multiplying by $u(s)-v_{h}(s)$

$$
\left\langle-\dot{u}_{h}(s), u(s)-v_{h}(s)\right\rangle+\left\langle-B\left(v_{h}(s)\right), u(s)-v_{h}(s)\right\rangle \leq\left\langle-f_{h}(s), u(s)-v_{h}(s)\right\rangle, \text { a.e. on }[0, T] .
$$

By summing (3.6) and (3.7) we obtain

$$
\begin{array}{r}
\left\langle\dot{u}-\dot{u}_{h}, u-u_{h}\right\rangle+\left\langle B(u)-B\left(u_{h}\right), u-u_{h}\right\rangle \leq-\left\langle B\left(u_{h}\right)-B\left(v_{h}\right), u-u_{h}\right\rangle \\
+\left\langle f-f_{h}, u-u_{h}\right\rangle+\left\langle f_{h}-\dot{u}_{h}-B\left(v_{h}\right), v_{h}-u_{h}\right\rangle .
\end{array}
$$

We denote

$$
w=u-u_{h} .
$$

By integrating and using Cauchy-Schwarz's inequality, we obtain, thanks to assumptions (1.2a) and (1.2b)

$$
\begin{aligned}
\frac{1}{2}\left|w_{h}(t)\right|^{2}+\alpha \int_{0}^{t}\left\|w_{h}(s)\right\|^{2} \mathrm{~d} s \leq & l \int_{0}^{t}\left\|u_{h}(s)-v_{h}(s)\right\|\left\|w_{h}(s)\right\| \mathrm{d} s+\int_{0}^{t}\left\|f(s, u(s))-f_{h}(s)\right\|^{\prime}\left\|w_{h}(s)\right\| \mathrm{d} s \\
& +\int_{0}^{t}\left\langle f_{h}(s)-\dot{u}_{h}(s)-B\left(v_{h}(s)\right), v_{h}(s)-u_{h}(s)\right\rangle \mathrm{d} s
\end{aligned}
$$


As we did in the previous proofs, each product is bounded so that we can write

$$
\begin{aligned}
\left|w_{h}(t)\right|^{2}+\alpha \int_{0}^{t}\left\|w_{h}(s)\right\|^{2} \mathrm{~d} s \leq & \frac{2 l^{2}}{\alpha} \int_{0}^{T}\left\|u_{h}(s)-v_{h}(s)\right\|^{2} \mathrm{~d} s+\frac{2}{\alpha} \int_{0}^{t}\left(\left\|f(s, u(s))-f_{h}(s)\right\|^{\prime}\right)^{2} \mathrm{~d} s \\
& +2 \int_{0}^{t}\left\langle f_{h}(s)-\dot{u}_{h}(s)-B\left(v_{h}(s)\right), v_{h}(s)-u_{h}(s)\right\rangle \mathrm{d} s .
\end{aligned}
$$

We have otherwise, according to assumption (1.2c)

$$
\begin{aligned}
& \int_{0}^{t}\left(\left\|f(s, u(s))-f_{h}(s)\right\|^{\prime}\right)^{2} \mathrm{~d} s \leq 3\left(L^{2} \int_{0}^{t}\left|u(s)-u_{h}(s)\right|^{2} \mathrm{~d} s+L^{2} \int_{0}^{T}\left|u_{h}(s)-\widetilde{v}_{h}(s)\right|^{2} \mathrm{~d} s\right. \\
&\left.+\int_{0}^{T}\left(\left\|f\left(s, \widetilde{v}_{h}(s)\right)-f_{h}(s)\right\|^{\prime}\right)^{2} \mathrm{~d} s\right)
\end{aligned}
$$

and, according to Lemma 2.3 and 2.4 , this is bounded by

$$
3 L^{2} \int_{0}^{t}\left|u(s)-u_{h}(s)\right|^{2} \mathrm{~d} s+3\left(L^{2} M_{1}^{2}+M_{2}^{2}\right) h^{2}
$$

Similarly,

$$
\int_{0}^{T}\left\|u_{h}(s)-v_{h}(s)\right\|^{2} \mathrm{~d} s \leq M_{2}^{2} h^{2}
$$

We can then infer from (3.8) that there exists $C_{1}$ and $C_{2}$ not depending on $N$ such that

$$
\begin{aligned}
& \forall t \in[0, T], \quad\left|w_{h}(t)\right|^{2}+\alpha \int_{0}^{t}\left\|w_{h}(s)\right\|^{2} \mathrm{~d} s \leq C_{1} h^{2}+C_{2} \int_{0}^{t}\left|w_{h}(s)\right|^{2} \mathrm{~d} s \\
& +2 \int_{0}^{t}\left\langle f_{h}(s)-\dot{u}_{h}(s)-B\left(v_{h}(s)\right), v_{h}(s)-u_{h}(s)\right\rangle \mathrm{d} s .
\end{aligned}
$$

We conclude thanks to the Gronwall's lemma and the following lemma (whose proof is inspired by Lippold [16]).

Lemma 3.2. There exits a constant $C$ such that

$$
\forall N \in \mathbb{N}^{*}, \quad \forall t \in[0, T], \quad \int_{0}^{t}\left\langle f_{h}(s)-\dot{u}_{h}(s)-B\left(v_{h}(s)\right), v_{h}(s)-u_{h}(s)\right\rangle \mathrm{d} s \leq C h^{2} .
$$

Proof. Let us first prove that the integrand of (3.9) is non negative; indeed, on the interval $] t_{p}, t_{p+1}[$, it is equal to

$$
h\left(1-\frac{s-t_{p}}{h}\right)\left\langle f\left(t_{p}, U^{p}\right)-V^{p}-B\left(U^{p+1}\right), V^{p}\right\rangle
$$

Thanks to (3.1), relation (1.5) is equivalent to

$$
\forall p \in\{0, \ldots, N-1\}, \quad \forall z \in K, \quad\left\langle f\left(t_{p}, U^{p}\right)-V^{p}-B\left(U^{p+1}\right), U^{p+1}-z\right\rangle \geq 0 .
$$


If we substitute $z=U^{p}$ in (3.10), we infer immediately the desired positivity. Therefore, it is enough to estimate from above

$$
\int_{0}^{T}\left\langle f_{h}(s)-\dot{u}_{h}(s)-B\left(v_{h}(s)\right), v_{h}(s)-u_{h}(s)\right\rangle \mathrm{d} s
$$

which is equal to

$$
\frac{h^{2}}{2} \sum_{p=0}^{N-1}\left\langle f\left(t_{p}, U^{p}\right)-V^{p}-B\left(U^{p+1}\right), V^{p}\right\rangle .
$$

We will show now that the sum in (3.11) is bounded independently of $h$; for that purpose, we will decompose this sum into a part that can be estimated thanks to previous results and a telescopic part that will sum up to a simple expression. Let us set $z=U^{p+2}$ in (3.10); then, changing $p$ into $p-1$, we obtain the following inequality for $p \in\{1, \ldots, N-1\}$ :

$$
\left\langle f\left(t_{p-1}, U^{p-1}\right)-V^{p-1}-B\left(U^{p}\right), V^{p}\right\rangle \leq 0 .
$$

We rewrite the summand in (3.11) as

$\left\langle f\left(t_{p}, U^{p}\right)-V^{p}-B\left(U^{p+1}\right)-f\left(t_{p-1}, U^{p-1}\right)+V^{p-1}+B\left(U^{p}\right), V^{p}\right\rangle+\left\langle f\left(t_{p-1}, U^{p-1}\right)-V^{p-1}-B\left(U^{p}\right), V^{p}\right\rangle$.

Thanks to (3.12), it suffices now to estimate

$$
\begin{aligned}
\sum_{p=1}^{N-1}\left\langle f\left(t_{p}, U^{p}\right)-B\left(U^{p+1}\right)-f\left(t_{p-1}, U^{p-1}\right)\right. & \left.B\left(U^{p}\right), V^{p}\right\rangle \\
& +\sum_{p=1}^{N-1}\left\langle V^{p-1}-V^{p}, V^{p}\right\rangle+\left\langle f\left(0, u_{0}\right)-V^{0}-B\left(U^{1}\right), V^{0}\right\rangle .
\end{aligned}
$$

Recalling the definition (2.16) of $\delta_{p}$ and by using assumption (1.2b) and (1.2c), the first summands in (3.13) are bounded by

$$
\left\|V^{p}\right\|\left(L h\left|V^{p-1}\right|+\left\|\delta_{p}\right\|^{\prime}\right) .
$$

Therefore,

$$
\begin{aligned}
& \sum_{p=1}^{N-1}\left\langle f\left(t_{p}, U^{p}\right)-B\left(U^{p+1}\right)-f\left(t_{p-1}, U^{p-1}\right)+B\left(U^{p}\right), V^{p}\right\rangle \\
& \leq L h \sum_{p=1}^{N-1}\left\|V^{p}\right\|\left|V^{p-1}\right|+\left(h \sum_{p=1}^{N-1}\left\|V^{p}\right\|^{2}\right)^{1 / 2}\left(\sum_{p=1}^{N-1} \frac{\left\|\delta_{p}\right\|^{2}}{h}\right)^{1 / 2}, \\
& \leq L\left(\max \left|V^{p-1}\right|\right)\left(T \sum_{p=1}^{N-1} h\left\|V^{p}\right\|^{2}\right)^{1 / 2}+\left(h \sum_{p=1}^{N-1}\left\|V^{p}\right\|^{2}\right)^{1 / 2}\left(\sum_{p=1}^{N-1} \frac{\left\|\delta_{p}\right\|^{2}}{h}\right)^{1 / 2} .
\end{aligned}
$$


According to (2.5), (2.6) and (2.17), the right hand side of above expression is bounded uniformly in $p$ and $h$. The second sum in (3.13) is estimated by

$$
\sum_{p=1}^{N-1}\left(\frac{\left|V^{p-1}\right|^{2}+\left|V^{p}\right|^{2}}{2}\right)-\sum_{p=1}^{N-1}\left|V^{p}\right|^{2} \leq \frac{\left|V^{0}\right|^{2}}{2} \leq \frac{M^{2}}{2},
$$

according to (2.5). The last term in (3.13) is estimated thanks to assumption (1.2e) which implies that there exits $w \in H$ such that

$$
\left\langle f\left(0, u_{0}\right)-w-B\left(U^{0}\right), V^{0}\right\rangle \geq 0
$$

since $0 \in A\left(U^{1}\right)$ and, thanks to coercivity of $B$,

$$
\begin{aligned}
\left\langle f\left(0, u_{0}\right)-V^{0}-B\left(U^{1}\right), V^{0}\right\rangle & \leq\left\langle w-V^{0}, V^{0}\right\rangle+\left\langle B\left(U^{0}\right)-B\left(U^{1}\right), V^{0}\right\rangle, \\
& \leq\left\langle w-V^{0}, V^{0}\right\rangle, \\
& \leq|w|\left|V^{0}\right|, \\
& \leq M|w|,
\end{aligned}
$$

and hence the conclusion.

\section{Other Results of COnVERGence When $V=H=V^{\prime}$}

In the mechanical application presented in Section 7, the operator $B$ is not coercive. Our theory applies to non coercitive $B$ if $V=H=V^{\prime}$; we assume that (1.2a) holds; assumption (1.2b) is not necessary anymore and (1.2c) and (1.2d) hold. Assumption (1.2e) holds automatically. The differential inclusion (1.3) is equivalent to

$$
\dot{u}+A(u) \ni g(., u),
$$

where $g(t, u)=f(t, u)-B(u)$. The function $g$ has the same properties as $f$. Thus, we will study in this section the convergence of the numerical scheme (1.19) to the solution of the inclusion (1.20) under assumptions (1.2c), (1.2d) and (1.18).

Let us review briefly the modifications of the results of Sections 2 and 3 needed for the proofs.

The uniqueness is straightforward.

Under assumptions (1.18), (1.2c) and (1.2d), Lemma 2.2 is replaced by

Lemma 4.1. There exists a constant $M$ such that, for all $N \in \mathbb{N}^{*}$,

$$
\begin{aligned}
& \forall p \in\{0, \ldots, N\}, \quad\left|U^{p}-u_{0}\right| \leq M \\
& \forall p \in\{0, \ldots, N-1\}, \quad \frac{1}{h}\left|U^{p+1}-U^{p}\right| \leq M
\end{aligned}
$$

Proof. In order to prove (4.1), we use again the estimate proved at the beginning of the proof of Lemma 2.2 where the constant $\alpha$ and function $B$ are set equal to zero. As in that proof, we prove, by using the classical inequality (2.2) and relation (2.10)

$$
\left(1-\frac{h}{2 T}\right)\left|U^{p+1}-u_{0}\right|^{2} \leq\left(1+4 L^{2} T h\right)\left|U^{p}-u_{0}\right|^{2}+4 T h\left|f\left(t_{p}, u_{0}\right)-f\left(0, u_{0}\right)-w\right|^{2} .
$$


Since for all $h \in] 0, T]$, we have

$$
0<\left(1-\frac{h}{2 T}\right)^{-1} \leq 1+\frac{5}{2 T} h
$$

we conclude as we did in the proof of Lemma 2.2 using the discrete Gronwall's lemma.

Estimate (4.2) is left to the reader.

For what follows, it is enough to estimate $\left\|u-u_{h}\right\|_{C^{0}([0, T], H)}$. Lemmas 2.3 and 2.4 are valid and we obtain

$$
\left\|u_{h}-v_{h}\right\|_{L^{2}(0, T ; H)}+\left\|u_{h}-\widetilde{v}_{h}\right\|_{L^{2}(0, T ; H)} \leq M_{1} h,
$$

and

$$
\left\|f\left(., \widetilde{v}_{h}\right)-f_{h}\right\|_{L^{2}(0, T ; H)} \leq M_{2} h .
$$

Proposition 2.5 is modified and simplified as follows:

Proposition 4.2. If assumptions (1.2c), (1.2d) and (1.18), hold, there exists a constant $C$ such that for all $h$, $k$ and all $t$ in $[0, T]$ :

$$
\left|u_{h}(t)-u_{k}(t)\right| \leq C \sqrt{h+k} .
$$

Proof. We set $\alpha=0$ in $(2.27)$, and by integration over $[0, t]$ we obtain the inequality

$$
\frac{1}{2}\left|u_{h}(t)-u_{k}(t)\right|^{2} \leq \int_{0}^{t}\left(\dot{u}_{h}-\dot{u}_{k}, v_{k}-v_{h}+u_{h}-u_{k}\right) \mathrm{d} s+\int_{0}^{t}\left\langle f_{h}-f_{k}, v_{h}-v_{k}\right\rangle .
$$

Each term of the right hand side of (4.7) is treated exactly as in the proof of Proposition 2.5, and we obtain

$$
\left|u_{h}(t)-u_{k}(t)\right|^{2} \leq M_{5}\left(h+k+\int_{0}^{t}\left|u_{h}-u_{k}\right|^{2} \mathrm{~d} s\right)
$$

where $M_{5}$ is independent of $h$ and $k$. We conclude with the help of Gronwall's Lemma.

We state now the existence result: indeed, thanks to Proposition $4.2\left(u_{h}\right)$ is a Cauchy sequence; results analogous to those of Propositions 2.6 and 2.7 can be proved; we summarize them as follows:

Proposition 4.3. Assume that (1.2c), (1.2d) and (1.18) hold. There exists a unique solution $u$ of (1.20) in $W^{1, \infty}(0, T ; H)$. Moreover, we have

$$
\lim _{h \rightarrow 0^{+}} u_{h}=u \text { in } C^{0}([0, T], H) \text {. }
$$

Proposition 4.4. Under assumptions (1.2c), (1.2d) and (1.18), there exists $C$ such that, for all $h>0$,

$$
\forall t \in[0, T], \quad\left|u(t)-u_{h}(t)\right| \leq C \sqrt{h} .
$$

Similarly to Proposition 3.1, we prove the order one of the scheme.

Proposition 4.5. Let $K$ be a convex non empty closed subset of $H$ and let $A$ be the maximal monotone operator $\partial \psi_{K}$. Under assumptions (1.2c), (1.2d) and (1.18), there exists $C$ such that, for all $h>0$,

$$
\forall t \in[0, T], \quad\left|u(t)-u_{h}(t)\right| \leq C h .
$$




\section{ORDER ONE IN FINITE DIMENSION FOR $K$ A PRODUCT OF $n$ INTERVALS}

We now assume that $H=l_{n}^{q}$, i.e. the space $\mathbb{R}^{n}$ equipped with the norm defined by (1.21). We assume that (1.22), (1.23) and (1.24) hold, where each $K_{i}$ is a non empty closed interval of $\mathbb{R}$. In finite dimension, $V=H=V^{\prime}$ and we keep the frame of Proposition 4.5; thus all the results of Section 4 are valid. In this section, we prove that the solution of the scheme

$$
\begin{aligned}
& \forall p \in\{0, \ldots, N-1\}, \quad \frac{U^{p+1}-U^{p}}{h}+\partial \psi_{K}\left(U^{p+1}\right) \ni f\left(t_{p}, U^{p}\right), \\
& U^{0}=u_{0}
\end{aligned}
$$

converges to the solution of the differential inclusion

$$
\begin{aligned}
& \left.\dot{u}(t)+\partial \psi_{K}(u(t)) \ni f(t, u(t)), \text { a.e. on }\right] 0, T[ \\
& u(0)=u_{0},
\end{aligned}
$$

with a precision one.

For all $N \in \mathbb{N}^{*}$ and for all $p \in\{0, \ldots, N-1\}$, we define $v^{p} \in W^{1, \infty}\left(t_{p}, T ; \mathbb{R}^{n}\right)$ as the solution of the problem

$$
\begin{aligned}
& \left.\dot{v}^{p}(t)+\partial \psi_{K}\left(v^{p}(t)\right) \ni f\left(t, v^{p}(t)\right), \text { a.e. on }\right] t_{p}, T[ \\
& v^{p}\left(t_{p}\right)=U^{p}
\end{aligned}
$$

Here is the strategie in order to prove Proposition 5.6: the main point consists in showing that the difference between $U^{p+1}$ and $v^{p}\left(t_{p+1}\right)$ is quadratic in $h$; this is a delicate result which depends strongly on assumption (1.22): this assumption let us decouple the problem as sequence of $n$-dimensional problems; we first prove that the error is quadratic when the convex is a half line; this proof (Prop. 5.4) entails the treatment of four cases; it is suitably modified (Prop. 5.5) to encompass the case of an interval.

In order to obtain this quadratic estimate on the error, we show at Lemma 5.1 an estimate on $\left\|\dot{v}_{p}\right\|_{L^{\infty}\left(t_{p}, t_{p+1} ; l_{n}^{q}\right)}$ where we emphasize the dependence of the constants of the problem; we deduce at Corollary 5.2 that $f\left(., v^{p}().\right)$ has a Lipschitz constant independent of $N$.

Lemma 5.1. Under hypotheses (1.22), (1.23) and (1.24), the derivative $\dot{v}_{p}$ is bounded in $L^{\infty}\left(t_{p}, t_{p+1} ; l_{n}^{q}\right)$ independently of $p$ and $N$.

Proof. According to Proposition 3.4 of [6], equation (5.5) implies

$$
\dot{v}^{p}(t+0)=f\left(t, v^{p}(t)\right)-\operatorname{proj}_{\partial \psi_{K}\left(v^{p}(t)\right)}\left(f\left(t, v^{p}(t)\right)\right),
$$

since $f$ is smooth enough for that result to hold. Let $\left(e_{i}\right)_{1 \leq i \leq n}$ be the canonical basis of $\mathbb{R}^{n}$; we set

$$
\begin{aligned}
& \forall t \in[0, T], \quad \forall x \in \mathbb{R}^{n}, \quad f(t, x)=\sum_{i=1}^{n} f_{i}(t, x) e_{i}, \\
& \forall t \in[0, T], \quad v^{p}(t)=\sum_{i=1}^{n} v_{i}^{p}(t) e_{i} .
\end{aligned}
$$

We have then the componentwise relation

$$
\dot{v}_{i}^{p}(t+0)=f_{i}\left(t, v^{p}(t)\right)-\operatorname{proj}_{\partial \psi_{K_{i}}\left(v_{i}^{p}(t)\right)}\left(f_{i}\left(t, v^{p}(t)\right)\right)
$$


which implies that for all $i=1, \ldots, n$, since the section of $\partial \psi_{K_{i}}$ vanishes,

$$
\left|\dot{v}_{i}^{p}(t+0)\right| \leq 2\left|f_{i}\left(t, v^{p}(t)\right)\right|
$$

and, hence, for all $q \in[1,+\infty]$

$$
\left|\dot{v}^{p}(t+0)\right|_{q} \leq 2\left|f\left(t, v^{p}(t)\right)\right|_{q}
$$

We use the Lipschitz property of $f$ and the triangle inequality to derive an estimate on $\dot{v}_{p}$

$$
\left|\dot{v}^{p}(t+0)\right|_{q} \leq 2 L\left(\left|v^{p}(t)-U^{p}\right|_{q}+\left|U^{p}-u_{0}\right|_{q}\right)+2\left|f\left(t, u_{0}\right)\right|_{q} .
$$

By integrating over $\left[t_{p}, t\right],(5.8)$ is converted into a Gronwall inequality which implies immediately for $t \in$ $\left[t_{p}, t_{p+1}\right]$

$$
\left|v^{p}(t)-U^{p}\right|_{q} \leq 2 \mathrm{e}^{2 L h}\left(\left|f\left(., u_{0}\right)\right|_{L^{\infty}\left(0, T ; l_{n}^{q}\right)}+L\left|U^{p}-u_{0}\right|\right),
$$

and hence the conclusion, thanks to (5.8).

Corollary 5.2. Under hypotheses (1.22), (1.23) and (1.24), there exists $M$ such that, for all $N \in \mathbb{N}^{*}$, for all $p \in\{0, \ldots, N-1\}$,

$$
\forall \tau_{1}, \tau_{2} \in\left[t_{p}, t_{p+1}\right], \quad\left|f\left(\tau_{1}, v^{p}\left(\tau_{1}\right)\right)-f\left(\tau_{2}, v^{p}\left(\tau_{2}\right)\right)\right|_{q} \leq M\left|\tau_{2}-\tau_{1}\right| .
$$

Proof. This is an immediate consequence of Lemma 5.1, since we have, for all $\tau_{1}, \tau_{2} \in\left[t_{p}, t_{p+1}\right]$,

$$
\left|f_{i}\left(\tau_{1}, v^{p}\left(\tau_{1}\right)\right)-f_{i}\left(\tau_{2}, v^{p}\left(\tau_{2}\right)\right)\right| \leq\left|f_{i}\left(\tau_{1}, v^{p}\left(\tau_{1}\right)\right)-f_{i}\left(\tau_{1}, v^{p}\left(\tau_{2}\right)\right)\right|+\left|\int_{\tau_{1}}^{\tau_{2}} \frac{\partial f_{i}}{\partial t}\left(s, v_{p}\left(\tau_{2}\right)\right) \mathrm{d} s\right|,
$$

which implies

$$
\left|f\left(\tau_{1}, v^{p}\left(\tau_{1}\right)\right)-f\left(\tau_{2}, v^{p}\left(\tau_{2}\right)\right)\right|_{q} \leq L \int_{\tau_{1}}^{\tau_{2}}\left|\dot{v}_{p}(s)\right|_{q} \mathrm{~d} s+\int_{\tau_{1}}^{\tau_{2}}\left|\frac{\partial f}{\partial t}\left(s, v_{p}\left(\tau_{2}\right)\right)\right|_{q} \mathrm{~d} s .
$$

Before we prove Proposition 5.6, we will give a lemma which permits us to determine values of the $i$-th component of the right hand side of (5.5) rewritten as

$$
\dot{v}_{i}^{p}(t)+\partial \psi_{K_{i}}\left(v_{i}^{p}(t)\right) \ni f_{i}\left(t, v^{p}(t)\right),
$$

if $v_{i}^{p}(t)$ is equal to zero:

Lemma 5.3. Let $a, b, \tau \in \mathbb{R}$ such that $a<\tau<b$, let $w \in W^{1, \infty}(a, b)$ and let $g$ be a continuous function such that

$$
\left.\dot{w}(t)+\partial \psi_{\mathbb{R}_{+}}(w(t)) \ni g(t) \text { a.e. on }\right] a, b[.
$$

If $w(\tau)=0$, then $g(\tau) \leq 0$; moreover, if $g(\tau)<0$, then there exists an interval $] \tau, \tau^{\prime}[$ where $w$ vanishes. 
Proof. Assume $g(\tau)>0$; there exists an interval $] a^{\prime}, b^{\prime}[\subset] a, b[$, including $\tau$ such that $g(s) \geq g(\tau) / 2$ over $] a^{\prime}, b^{\prime}[$. According to Proposition 3.4 p. 69 of [6], we have

$$
\forall t \in] a, b\left[, \quad \dot{w}(t+0)= \begin{cases}g^{+}(t), & \text { si } w(t)=0 \\ g(t), & \text { si } w(t)>0\end{cases}\right.
$$

where $g^{+}(t)$ is the positive part of $g(t)$. Thus, we can integrate the inequality on $] a^{\prime}, b^{\prime}[$

$$
\forall t \in] a^{\prime}, b^{\prime}\left[, \quad \dot{w}(t+0) \geq \frac{1}{2} g(\tau),\right.
$$

which implies

$$
w(\tau) \geq w\left(a^{\prime}\right)+\frac{1}{2} g(\tau)\left(\tau-a^{\prime}\right)>0,
$$

which contradicts $w(\tau)=0$.

If $g(\tau)<0$, then there exists a maximal interval $] \tau, \tau^{\prime}[\subset] a, b[$ on which $g$ is strictly negative. We remark that the zero function $] \tau, \tau^{\prime}$ [ solves (5.11); since it had the same vanishing initial condition as $w$ at $\tau$ by uniqueness $w$ vanishes over $] \tau, \tau^{\prime}[$.

We set

$$
\forall p \in\{0, \ldots, N\}, \quad U^{p}=\sum_{i=1}^{n} U_{i}^{p} e_{i}
$$

In order to show an estimate on $\left|v^{p}\left(t_{p+1}\right)-U^{p+1}\right|_{q}$, it is enough to estimate $\left|v_{i}^{p}\left(t_{p+1}\right)-U_{i}^{p+1}\right|$; this is the object of two following propositions:

Proposition 5.4. Let $i$ be in $\{1, \ldots, n\}$. Under hypothesis (1.22), (1.23) and (1.24) and if $K_{i}=\mathbb{R}_{+}$, there exists $M_{i}$, independently of $i, N$ and $p$ such that

$$
\left|v_{i}^{p}\left(t_{p+1}\right)-U_{i}^{p+1}\right| \leq M_{i} h^{2} .
$$

Proof. With respect to [6], for all convex non empty closed subset $K$ of $\mathbb{R}^{n}$, and for all $\lambda>0,\left(\mathbf{1}+\lambda \partial \psi_{K}\right)^{-1}=$ $\operatorname{proj}_{K}$; we rewrite then (5.1) under the form

$$
U_{i}^{p+1}=\left(h f_{i}\left(t_{p}, U^{p}\right)+U_{i}^{p}\right)^{+} .
$$

We set

$$
J_{i}=\{t \in] t_{p}, t_{p+1}\left[: v_{i}^{p}(t)=0\right\}
$$

If $J_{i}$ is empty

$$
\left.\dot{v}_{i}^{p}(t)=f_{i}\left(t, v^{p}(t)\right), \quad \text { a.e. on }\right] t_{p}, t_{p+1}[.
$$

We combine the alternatives $J_{i}=\emptyset$ or $J_{i} \neq \emptyset$ and $U_{i}^{p+1}>0$ or $U_{i}^{p+1}=0$ to get four cases, which we study in turn. 
First case: $J_{i}=\emptyset$ and $U_{i}^{p+1}>0$.

According to (5.12) and (5.13),

$$
\left|v_{i}^{p}\left(t_{p+1}\right)-U_{i}^{p+1}\right|=\left|\int_{t_{p}}^{t_{p+1}} f_{i}\left(s, v^{p}(s)\right)-f_{i}\left(t_{p}, U^{p}\right) \mathrm{d} s\right|=\left|\int_{t_{p}}^{t_{p+1}} f_{i}\left(s, v^{p}(s)\right)-f_{i}\left(t_{p}, v^{p}\left(t_{p}\right)\right) \mathrm{d} s\right|,
$$

which implies thanks to Corollary 5.2,

$$
\left|v_{i}^{p}\left(t_{p+1}\right)-U_{i}^{p+1}\right| \leq M h^{2}
$$

Second case: $J_{i}=\emptyset$ and $U_{i}^{p+1}=0$.

Since $v_{i}^{p}\left(t_{p+1}\right)$ is non negative, we obtain, according to (5.13),

$$
\left|v_{i}^{p}\left(t_{p+1}\right)-U_{i}^{p+1}\right|=\int_{t_{p}}^{t_{p+1}} f_{i}\left(s, v^{p}(s)\right) \mathrm{d} s+U_{i}^{p}
$$

Since $U_{i}^{p+1}=\left(h f_{i}\left(t_{p}, U^{p}\right)+U_{i}^{p}\right)^{+}$vanishes, we have

$$
U_{i}^{p} \leq-h f_{i}\left(t_{p}, U^{p}\right),
$$

and

$$
\left|v_{i}^{p}\left(t_{p+1}\right)-U_{i}^{p+1}\right| \leq \int_{t_{p}}^{t_{p+1}} f_{i}\left(s, v^{p}(s)\right)-f_{i}\left(t_{p}, U^{p}\right) \mathrm{d} s
$$

We conclude as above.

Third case: $J_{i} \neq \emptyset$ and $U_{i}^{p+1}>0$. We consider again two cases with respect to the value of $v_{i}^{p}\left(t_{p+1}\right)$.

First subcase: $v_{i}^{p}\left(t_{p+1}\right)>0$.

Let $\tau=\inf \left(J_{i}\right) \in\left[t_{p}, t_{p+1}\left[\right.\right.$ and $\left.\tau^{\prime}=\sup \left(J_{i}\right) \in\right] t_{p}, t_{p+1}$ [ be the first and the last zero of $v_{i}^{p}$ in $\left[t_{p}, t_{p+1}\right]$. By integrating the function $\dot{v}_{i}^{p}$ over $\left[t_{p}, \tau\right]$ and $\left[\tau^{\prime}, t_{p+1}\right]$, we obtain

$$
-U_{i}^{p}=\int_{t_{p}}^{\tau} f_{i}\left(s, v^{p}(s)\right) \mathrm{d} s \text { and } v_{i}^{p}\left(t_{p+1}\right)=\int_{\tau^{\prime}}^{t_{p+1}} f_{i}\left(s, v^{p}(s)\right) \mathrm{d} s .
$$

Since $U_{i}^{p+1}=\left(h_{i} f\left(t_{p}, U^{p}\right)+U_{i}^{p}\right)^{+}$is strictly positive, we have

$$
\begin{aligned}
v_{i}^{p}\left(t_{p+1}\right)-U_{i}^{p+1} & =\int_{\tau^{\prime}}^{t_{p+1}} f_{i}\left(s, v^{p}(s)\right) \mathrm{d} s-h f_{i}\left(t_{p}, U^{p}\right)-U_{i}^{p}, \\
& =\int_{\tau^{\prime}}^{t_{p+1}} f_{i}\left(s, v^{p}(s)\right) \mathrm{d} s-h f_{i}\left(t_{p}, U^{p}\right)+\int_{t_{p}}^{\tau} f_{i}\left(s, v^{p}(s)\right) \mathrm{d} s .
\end{aligned}
$$

$\tau^{\prime}<t_{p+1}$, because $v_{i}^{p}\left(t_{p+1}\right)>0$ and since $v_{i}^{p}$ is strictly positive on $\left.] \tau^{\prime}, t_{p+1}\right]$ and the function $f_{i}\left(., v^{p}\right)$ is continuous, Lemma 5.3 implies that $f_{i}\left(\tau^{\prime}, v^{p}\left(\tau^{\prime}\right)\right)$ vanishes; thanks to Corollary 5.2, we obtain

$$
\forall t \in\left[t_{p}, t_{p+1}\right], \quad\left|f_{i}\left(t, v^{p}(t)\right)\right| \leq M h,
$$


and

$$
\left|v_{i}^{p}\left(t_{p+1}\right)-U_{i}^{p+1}\right| \leq M h\left(t_{p+1}-\tau^{\prime}+h+\tau-t_{p}\right)
$$

which implies

$$
\left|v_{i}^{p}\left(t_{p+1}\right)-U_{i}^{p+1}\right| \leq 2 M h^{2}
$$

Second subcase: $v_{i}^{p}\left(t_{p+1}\right)=0$.

In this case, $\tau^{\prime}$ can be equal to $t_{p+1}$ and we cannot apply Lemma 5.3. Let $w_{i}^{p} \in W^{1, \infty}\left(t_{p}, t_{p+1}\right)$ be the solution of the following problem, without constraint

$$
\begin{aligned}
& \left.\dot{w}_{i}^{p}(t)=f_{i}\left(t, v^{p}(t)\right), \text { a.e. on }\right] t_{p}, t_{p+1}[ \\
& w_{i}^{p}\left(t_{p}\right)=U_{i}^{p}
\end{aligned}
$$

Let us compare $w_{i}^{p}(t)$ to $v_{i}^{p}(t)$ by using (5.7), rewritten as

$$
\forall t \in\left[t_{p}, t_{p+1}\left[, \quad \dot{v}_{i}^{p}(t+0)= \begin{cases}f_{i}\left(t, v^{p}(t)\right), & \text { if } v_{i}^{p}(t)>0 \\ f_{i}^{+}\left(t, v^{p}(t)\right), & \text { if } v_{i}^{p}(t)=0\end{cases}\right.\right.
$$

Then,

$$
\forall t \in\left[t_{p}, t_{p+1}\left[, \quad \dot{w}_{i}^{p}(t+0)-\dot{v}_{i}^{p}(t+0)= \begin{cases}0 & \text { if } v_{i}^{p}(t)>0 \\ f_{i}\left(t, v^{p}(t)\right)-f_{i}^{+}\left(t, v^{p}(t)\right)=-f_{i}^{-}\left(t, v^{p}(t)\right), & \text { if } v_{i}^{p}(t)=0\end{cases}\right.\right.
$$

This expression is always negative and, by integration, we obtain,

$$
\forall t \in\left[t_{p}, t_{p+1}\left[, \quad w_{i}^{p}(t)-v_{i}^{p}(t) \leq 0\right.\right.
$$

Thus, $w_{i}^{p}\left(t_{p+1}\right) \leq 0$ and we have

$$
\left|v_{i}^{p}\left(t_{p+1}\right)-U_{i}^{p+1}\right|=U_{i}^{p+1} \leq h f_{i}\left(t_{p}, U_{i}^{p}\right)+U_{i}^{p}-w_{i}^{p}\left(t_{p+1}\right)=h f_{i}\left(t_{p}, U_{i}^{p}\right)-\int_{t_{p}}^{t_{p+1}} f_{i}\left(s, v^{p}(s)\right) \mathrm{d} s,
$$

and we conclude as previously.

Fourth case: $J_{i} \neq \emptyset$ and $U_{i}^{p+1}=0$.

If $v_{i}^{p}\left(t_{p+1}\right)=0$, then the conclusion is immediate. If $v_{i}^{p}\left(t_{p+1}\right)>0$, we have, as in the first subcase of the third case

$$
\left.v_{i}^{p}\left(t_{p+1}\right)=\int_{\tau^{\prime}}^{t_{p+1}} f_{i}\left(s, v^{p}(s)\right) \mathrm{d} s, \quad \tau^{\prime} \in\right] t_{p}, t_{p+1}\left[\text { and } f_{i}\left(\tau^{\prime}, v^{p}\left(\tau^{\prime}\right)\right)=0,\right.
$$

which permits us to conclude as before because

$$
\left|v_{i}^{p}\left(t_{p+1}\right)-U_{i}^{p+1}\right|=\left|\int_{\tau^{\prime}}^{t_{p+1}} f_{i}\left(s, v^{p}(s)\right)-f_{i}\left(\tau^{\prime}, v^{p}\left(\tau^{\prime}\right)\right) \mathrm{d} s\right|
$$


Proposition 5.5. Let $i$ be in $\{1, \ldots, n\}$. Under hypothesis (1.22), (1.23) and (1.24) and if $K_{i}$ is a non empty closed interval of $\mathbb{R}$, there exists $M_{i}$ independently of $i, N$ and $p$ such that

$$
\left|v_{i}^{p}\left(t_{p+1}\right)-U_{i}^{p+1}\right| \leq M_{i} h^{2}
$$

Proof. If $K_{i}$ is equal to $\mathbb{R}$ or $[a,+\infty[$ or $]-\infty, a]$, the result is plain.

If $K_{i}=\{a\}$, then according to $(3.1) \partial \psi_{\{a\}}(a)=\mathbb{R} ;(5.1)$ can be rewritten under the form $U_{i}^{p+1}=a$ and according to $(5.10), v_{i}^{p}(t)=a$ on $\left[t_{p}, t_{p+1}[\right.$.

Assume now that $K_{i}=[a, b]$ where $a<b$. Here is how to modify the proof of Proposition 5.4 : if $K=[a, b]$, then (5.12) becomes

$$
U_{i}^{p+1}=\operatorname{proj}_{[a, b]}\left(h f_{i}\left(t_{p}, U^{p}\right)+U_{i}^{p}\right) .
$$

Set again

$$
J_{i}=\{t \in] t_{p}, t_{p+1}\left[: v_{i}^{p}(t)=a \text { or } v_{i}^{p}(t)=b\right\}
$$

We study again the four cases previously seen whether $J_{i}$ is empty or not and whether $U_{i}^{p+1}$ gains the values $a$ or $b$ or not.

First case: $J_{i}=\emptyset$ and $\left.U_{i}^{p+1} \in\right] a, b[$.

This case is similar to the first case of the proof of Proposition 5.4, because in this case we study problem without constraint.

Second case: $J_{i}=\emptyset$ and $U_{i}^{p+1} \in\{a, b\}$.

This case is similar to the second case of the proof of Proposition 5.4; indeed, if $U_{i}^{p+1}=a$, then $U_{i}^{p}-a \leq$ $-h f_{i}\left(t_{p}, U^{p}\right)$ and we obtain

$$
\begin{aligned}
\left|v_{i}^{p}\left(t_{p+1}\right)-U_{i}^{p+1}\right| & =v_{i}^{p}\left(t_{p+1}\right)-a \\
& =\int_{t_{p}}^{t_{p+1}} f_{i}\left(s, v^{p}(s)\right) \mathrm{d} s+U_{i}^{p}-a \\
& \leq \int_{t_{p}}^{t_{p+1}} f_{i}\left(s, v^{p}(s)\right)-f_{i}\left(t_{p}, U^{p}\right) \mathrm{d} s .
\end{aligned}
$$

Third case: $J_{i} \neq \emptyset$ and $\left.U_{i}^{p+1} \in\right] a, b[$.

We set

$$
J_{i}^{a}=\{t \in] t_{p}, t_{p+1}\left[: v_{i}^{p}(t)=a\right\} \text { and } J_{i}^{b}=\{t \in] t_{p}, t_{p+1}\left[: v_{i}^{p}(t)=b\right\}
$$

The case where $J_{i}^{a}$ or $J_{i}^{b}$ is empty reduces to the third case of the proof of Proposition 5.4, since contact takes place at one end only of $K_{i}$.

If $J_{i}^{a} \neq \emptyset$ and $J_{i}^{b} \neq \emptyset$, we can assume without loss of generality that there exists $\left.\theta \in\right] t_{p}, t_{p+1}\left[\right.$ and $\left.\theta^{\prime} \in\right] t_{p}, t_{p+1}[$ such that $\theta<\theta^{\prime}, v_{i}^{p}(\theta)=a$ and $v_{i}^{p}(\theta)=b$. Thus, by setting

$$
\theta_{1}=\sup \{t \in] \theta, \theta^{\prime}\left[: \quad v_{i}^{p}(t)=a\right\},
$$


we observe that $v_{i}^{p}\left(\theta_{1}\right)=a$ and there exists $\left.\theta_{2} \in\right] t_{p}, t_{p+1}[$ such that for all $t \in] \theta_{1}, \theta_{2}\left[, v_{i}^{p}(t)>a\right.$. Similarly to Lemma 5.3 , we can prove that $f_{i}\left(\theta_{1}, v^{p}\left(\theta_{1}\right)\right)$ vanishes; according to Corollary 5.2

$$
\forall t \in\left[t_{p}, t_{p+1}\right], \quad\left|f_{i}\left(t, v^{p}(t)\right)\right| \leq M h .
$$

We conclude as in the first subcase of the third case of Proposition 5.4: let $\tau=\inf \left(J_{i}\right) \in\left[t_{p}, t_{p+1}[\right.$ and $\left.\left.\tau^{\prime}=\sup \left(J_{i}\right) \in\right] t_{p}, t_{p+1}\right]$ be the first and the last zero of $v_{i}^{p}$ in $\left[t_{p}, t_{p+1}\right]$; we have

$$
v_{i}^{p}\left(t_{p+1}\right)-U_{i}^{p+1}=\int_{\tau^{\prime}}^{t_{p+1}} f_{i}\left(s, v^{p}(s)\right) \mathrm{d} s-h f_{i}\left(t_{p}, U^{p}\right)+\int_{t_{p}}^{\tau} f_{i}\left(s, v^{p}(s)\right) \mathrm{d} s .
$$

Fourth case: $J_{i} \neq \emptyset$ and $U_{i}^{p+1} \in\{a, b\}$.

If $v_{i}^{p}$ is strictly smaller than $b$ (resp. strictly greater than $a$ ) on $] t_{p}, t_{p+1}[$, the proof is similar to that of the fourth case of Proposition 5.4 since there is no contact at $b$ (resp. $a$ ).

Otherwise, we can assume that $v_{i}^{p}$ reaches at least once the value $a$ on $] t_{p}, t_{p+1}\left[\right.$ and that $U_{i}^{p+1}=b$. Since $\operatorname{proj}_{[a, b]}\left(h f_{i}\left(t_{p}, U^{p}\right)+U_{i}^{p}\right)=b$, we have $h f_{i}\left(t_{p}, U^{p}\right)+U_{i}^{p} \geq b$; furthermore, $v_{i}^{p}\left(t_{p+1}\right) \leq b$; thus

$$
\left|v_{i}^{p}\left(t_{p+1}\right)-U_{i}^{p+1}\right|=b-v_{i}^{p}\left(t_{p+1}\right) \leq h f_{i}\left(t_{p}, U^{p}\right)+U_{i}^{p}-v_{i}^{p}\left(t_{p+1}\right) .
$$

This reduces to the third case above since $v_{i}^{p}$ reaches at least once the value $a$ on $] t_{p}, t_{p+1}[$.

Proposition 5.6. If assumptions (1.22), (1.23) and (1.24) hold, there exists $C$ such that

$$
\forall N \in \mathbb{N}^{*}, \quad\left\|u-u_{h}\right\|_{C^{0}\left([0, T], l_{n}^{q}\right)} \leq C h .
$$

Proof. The $i$-th component of $v^{k}$ and $v^{k-1}$ satisfy respectively the equations

$$
\begin{aligned}
& \frac{\mathrm{d} v_{i}^{k}}{\mathrm{~d} t}+\partial \psi_{K_{i}}\left(v_{i}^{k}\right) \ni f_{i}\left(., v^{k}\right), \\
& \frac{\mathrm{d} v_{i}^{k-1}}{\mathrm{~d} t}+\partial \psi_{K_{i}}\left(v_{i}^{k-1}\right) \ni f_{i}\left(., v^{k-1}\right),
\end{aligned}
$$

over $\left[t_{k}, T\right]$. By monotonicity, we see that

$$
\frac{1}{2} \frac{\mathrm{d}}{\mathrm{d} t}\left|v_{i}^{k}-v_{i}^{k-1}\right|^{2} \leq\left|v_{i}^{k}-v_{i}^{k-1}\right|\left|f_{i}\left(., v^{k}\right)-f_{i}\left(., v^{k-1}\right)\right|,
$$

which implies

$$
\frac{\mathrm{d}}{\mathrm{d} t}\left|v_{i}^{k}-v_{i}^{k-1}\right| \leq\left|f_{i}\left(., v^{k}\right)-f_{i}\left(., v^{k-1}\right)\right|,
$$

and therefore by integrating over $\left[t_{k}, t\right]$

$$
\left|\left(v^{k}-v^{k-1}\right)(t)\right|_{q} \leq\left|\left(v^{k}-v^{k-1}\right)\left(t_{k}\right)\right|_{q}+\int_{t_{k}}^{t}\left|f\left(., v^{k}\right)-f\left(., v^{k-1}\right)\right|_{q} \mathrm{~d} s .
$$

This is a Gronwall inequality, from which we derive the estimate

$$
\left|\left(v^{k}-v^{k-1}\right)\left(t_{k}\right)\right|_{q} \leq \mathrm{e}^{L T} M h^{2},
$$


where we have used estimate (5.15) which implies

$$
\left|\left(v^{k}-v^{k-1}\right)\left(t_{k}\right)\right|_{q}=\left|U^{k}-v^{k-1}\left(t_{k}\right)\right|_{q} \leq M h^{2} .
$$

Therefore, because $v^{0}=u$,

$$
\begin{aligned}
\left|u_{h}\left(t_{p}\right)-u\left(t_{p}\right)\right|_{q} & \leq\left|U^{p}-v^{p}\left(t_{p}\right)\right|_{q}+\sum_{k=1}^{p}\left|v^{k}\left(t_{p}\right)-v^{k-1}\left(t_{p}\right)\right|_{q} \\
& \leq p M h^{2} \mathrm{e}^{L T} .
\end{aligned}
$$

Moreover, for $t \in\left[t_{p}, t_{p+1}\right]$, since the derivate of $u$ is integrable, we have

$$
\left|u_{h}(t)-u(t)\right|_{q} \leq C h .
$$

\section{Dependence on the DATA of THE ERRor ESTimate}

We are in the frame of the previous section. We will give now an explicit value of the constant $C$ of Proposition 5.6 in terms of the $n, q, L, T,\left\|f\left(., u_{0}\right)\right\|_{C^{0}\left([0, T], l_{n}^{q}\right)}$ and $\sup _{x \in G}\|\partial f / \partial t(., x)\|_{L^{\infty}\left(0, T ; l_{n}^{q}\right)}$ where $G$ is a bounded subset of $l_{n}^{q}$.

In order to simplify the following proposition, we set $n^{1 / q}=1$ if $q=+\infty$ and we define the function $P_{0}$ from $\mathbb{R}_{+} \times \mathbb{R}_{+}$to $\mathbb{R}_{+}$and the functions $P_{1}$ and $P_{2}$ from $\mathbb{N}^{*} \times[1,+\infty] \times \mathbb{R}_{+} \times \mathbb{R}_{+}$to $\mathbb{R}_{+}$by

$$
\begin{aligned}
P_{0}(L, T) & =4 \max (L T, 1) \mathrm{e}^{2 L T}, \\
P_{1}(n, q, L, T) & =96 n^{1 / q}(\max (L T, 1))^{3} \mathrm{e}^{4 L T}, \\
P_{2}(n, q, L, T) & =2 T n^{1 / q} \mathrm{e}^{L T} .
\end{aligned}
$$

Proposition 6.1. Assume that assumptions (1.22), (1.23) and (1.24) hold. Let $G$ be the bounded subset of $l_{n}^{q}$ defined by

$$
G=\left\{y \in l_{n}^{q}:\left|y-u_{0}\right|_{q} \leq\left\|f\left(., u_{0}\right)\right\|_{C^{0}\left([0, T], l_{n}^{q}\right)} P_{0}(L, T)\right\}
$$

Then

$$
\forall N \in \mathbb{N}^{*}, \quad\left\|u-u_{h}\right\|_{C^{0}\left([0, T], l_{n}^{q}\right)} \leq C h,
$$

where

$$
C=\left\|f\left(., u_{0}\right)\right\|_{C^{0}\left([0, T], l_{n}^{q}\right)} P_{1}(n, q, L, T)+\sup _{x \in G}\left\|\frac{\partial f}{\partial t}(., x)\right\|_{L^{\infty}\left(0, T ; l_{n}^{q}\right)} P_{2}(n, q, L, T) .
$$

Proof. The proof consists in walking trough the proofs of Section 5.

Let us give first an estimate of $\left\|u-u_{0}\right\|_{C^{0}\left([0, T], l_{n}^{q}\right)}$ and $\|\dot{u}\|_{L^{\infty}\left(0, T ; l_{n}^{q}\right)}$ in terms of data. As in (5.7), we obtain

$$
\forall t \in\left[0, T\left[, \quad\left|\dot{u}_{i}(t+0)\right| \leq 2\left|f_{i}(t, u(t))\right| .\right.\right.
$$

As in the beginning of the proof of Lemma 5.1, we obtain

$$
\left|u(t)-u_{0}\right|_{q} \leq 2 L \int_{0}^{t}\left|u(s)-u_{0}\right|_{q} \mathrm{~d} s+T\left\|f\left(., u_{0}\right)\right\|_{C^{0}\left([0, T], l_{n}^{q}\right)} .
$$


Thanks to the Gronwall's Lemma, we have

$$
\left\|u-u_{0}\right\|_{C^{0}\left([0, T], l_{n}^{q}\right)} \leq T\left\|f\left(., u_{0}\right)\right\|_{C^{0}\left([0, T], l_{n}^{q}\right)} \mathrm{e}^{2 L T},
$$

and according to $(6.3)$

$$
\|\dot{u}\|_{L^{\infty}\left(0, T ; l_{n}^{q}\right)} \leq 2\left\|f\left(., u_{0}\right)\right\|_{C^{0}\left([0, T], l_{n}^{q}\right)}\left(L T \mathrm{e}^{2 L T}+1\right) .
$$

The reader will then check that (6.2) can be deduced from (6.4).

Remark 6.2. The estimate of Proposition 6.1 does not depend on the set of constraints.

In the statement of Proposition 6.1, there is a term $n^{1 / q}$ which is unbounded as $n$ tends to infinity; indeed, in order to prove (5.17) we have summed the local errors componentwise (5.15). We can give a more accurate estimate without the $n^{1 / q}$ term if we replace our assumption (1.23) on $f$ by the following stronger hypothesis: for all $i$, there exists $L_{i} \in \mathbb{R}_{+}$such that

$$
\forall t \in[0, T], \quad \forall x_{1}, x_{2} \in \mathbb{R}^{n}, \quad\left|f_{i}\left(t, x_{1}\right)-f_{i}\left(t, x_{2}\right)\right| \leq L_{i}\left|x_{1}-x_{2}\right|_{q}
$$

We set

$$
L=\left|\left(L_{1}, \ldots, L_{n}\right)\right|_{q}
$$

If we define

$$
\begin{aligned}
& Q_{1}(L, T)=96(\max (L T, 1))^{3} \mathrm{e}^{4 L T} \\
& Q_{2}(L, T)=2 T \mathrm{e}^{L T}
\end{aligned}
$$

we can prove the following proposition:

Proposition 6.3. We assume that (1.22), (1.24), (6.5) and (6.6) hold. Let $G$ be the bounded set $l_{n}^{q}$ defined by (6.1). Then

$$
\forall N \in \mathbb{N}^{*}, \quad\left\|u-u_{h}\right\|_{C^{0}\left([0, T], l_{n}^{q}\right)} \leq C h
$$

where

$$
C=\left\|f\left(., u_{0}\right)\right\|_{C^{0}\left([0, T], l_{n}^{q}\right)} Q_{1}(L, T)+\sup _{x \in G}\left\|\frac{\partial f}{\partial t}(., x)\right\|_{L^{\infty}\left(0, T ; l_{n}^{q}\right)} Q_{2}(L, T) .
$$

Proof. We may apply here again the ideas of Proposition 6.1 and the reader will check the foregoing formula.

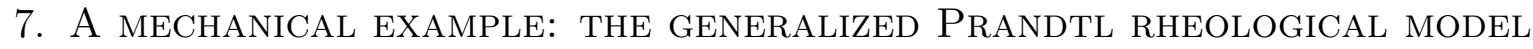

Let us review the Prandtl model with a finite number of degrees of freedom as presented in $[1,3]$ which motivated this work, since we observed the order one precision before proving them.

The Prandtl model consists of a material point connected with a finite parallel association of series associations made out of one spring and one dry friction or Saint-Venant element (see Fig. 2). Let $x$ be the abscissa of the material point, let $u_{i}$ be the displacement of the $i$-th spring (with stiffness $k_{i}$ ) and let $v_{i}$ be the displacement of the $i$-th Saint-Venant element (with threshold $\alpha_{i}$ ). The material point of mass $m$ is submitted to an external force $F$. Denote by $f_{i}$ the force exerted by the $i$-th spring. The constitutive law of the $i$-th spring is

$$
\tau_{i}=-k_{i} u_{i}
$$




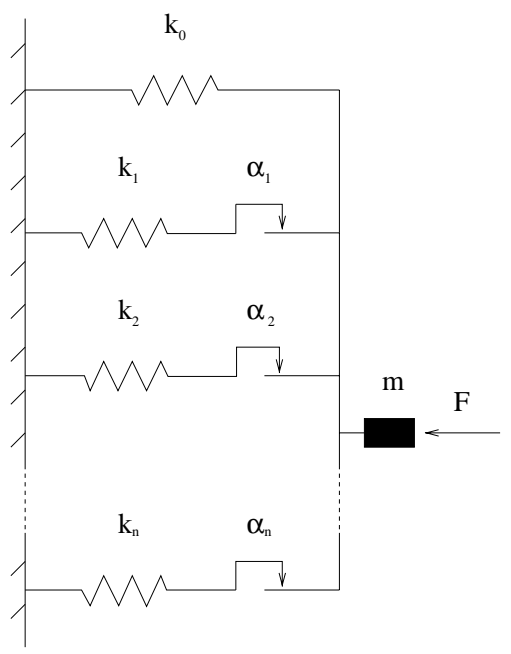

Figure 2. The generalized Prandtl rheological model with linear hardening.

We write the constitutive law of the $i$-th Saint-Venant element under the form

$$
\tau_{i} \in-\alpha_{i} \sigma\left(\dot{v}_{i}\right)
$$

where the maximal monotone graph $\sigma$ is given by

$$
\sigma(x)= \begin{cases}-1 & \text { if } x<0 \\ 1 & \text { if } x>0 \\ {[-1,1]} & \text { if } x=0\end{cases}
$$

The graph $\sigma$ is the inverse of the graph $\partial \psi_{[-1,1]}$ thus, (7.2) is equivalent to

$$
\dot{v}_{i} \in \partial \psi_{[-1,1]}\left(-\frac{\tau_{i}}{\alpha_{i}}\right) .
$$

Since we consider a parallel association of series associations, the total displacement of the series associations does not depend on $i$, that is

$$
\forall i \in\{1, \ldots, P\}, \quad x=u_{i}+v_{i}
$$

and the fundamental Theorem of dynamics gives

$$
m \ddot{x}=F-k_{0} x+\sum_{i=1}^{P} \tau_{i} .
$$

Define

$$
\forall i \in\{1, \ldots, P\}, \quad \eta_{i}=\alpha_{i} / k_{i}
$$


Equations (7.1), (7.3), (7.4) and (7.5) are equivalent to the following differential system:

$$
\begin{aligned}
& \dot{x}(t)=y(t), \text { a.e. on }[0, T], \\
& \dot{y}(t)=\frac{1}{m}\left(F(t)-k_{0} x(t)-\sum_{i=1}^{P} k_{i} u_{i}(t)\right), \text { a.e. on }[0, T], \\
& \forall i \in\{1, \ldots, P\}, \quad \dot{u}_{i}(t)+\partial \psi_{\left[-\eta_{i}, \eta_{i}\right]}\left(u_{i}(t)\right) \ni y(t), \text { a.e. on }[0, T],
\end{aligned}
$$

with initial conditions

$$
x(0)=x_{0}, \quad y(0)=y_{0}, \quad \forall i \in\{1, \ldots, P\}, \quad u_{i}(0)=u_{0, i} \in\left[-\eta_{i}, \eta_{i}\right] .
$$

The system (7.7) can be rewritten under the form

$$
\begin{aligned}
& \left.\dot{u}(t)+\partial \psi_{K}(u(t)) \ni f(t, u(t)), \text { a.e. on }\right] 0, T[, \\
& u(0)=u_{0},
\end{aligned}
$$

where the convex non empty closed subset $K$ of $\mathbb{R}^{n+2}$, the function $f$ from $[0, T] \times \mathbb{R}^{n+2}$ to $\mathbb{R}^{n+2}$, the function $u$ from $[0, T]$ to $\mathbb{R}^{n+2}$, the vector $u_{0} \in K$ are defined by

$$
\begin{aligned}
& K=\mathbb{R} \times \mathbb{R} \times\left[-\eta_{1}, \eta_{1}\right] \times \ldots \times\left[-\eta_{n}, \eta_{n}\right], \\
& f\left(t, x, y, u_{1}, \ldots, u_{n}\right)=\left(0, \frac{1}{m}\left(F(t)-k_{0} x-\sum_{i=1}^{n} k_{i} u_{i}\right), y, \ldots, y\right), \\
& u(t)=\left(x(t), y(t), u_{1}(t), \ldots, u_{n}(t)\right), \\
& u_{0}=\left(x_{0}, y_{0}, u_{1,0}, \ldots, u_{n, 0}\right) .
\end{aligned}
$$

In what follows, we assume that

$$
F \in W^{1, \infty}(0, T)
$$

By setting

$$
g(t)=\left(0, \frac{1}{m} F(t), 0, \ldots, 0\right)
$$

and

$$
C=\left(\begin{array}{ccccc}
0 & 0 & 0 & \ldots & 0 \\
k_{0} / m & 0 & k_{1} / m & \ldots & k_{n} / m \\
0 & -1 & 0 & \ldots & 0 \\
\vdots & \vdots & \vdots & \ldots & \vdots \\
0 & -1 & 0 & \ldots & 0
\end{array}\right)
$$

we can see that, for all $t \in[0, T]$, for all $X \in \mathbb{R}^{n+2}$

$$
f(t, X)=g(t)-C X,
$$

and

$$
\sup _{x \in \mathbb{R}^{n+2}}\left\|\frac{\partial f}{\partial t}(., x)\right\|_{L^{\infty}\left(0, T ; l_{n+2}^{q}\right)}=\|\dot{F}\|_{L^{\infty}(0, T)}
$$


Thus assumptions (1.2c), (1.2d) and (1.18) hold with

$$
L=\left|\|C \mid\|_{2},\right.
$$

where $\||C| \mid\|_{2}$ denote the operator norm subordinate to the $l_{n}^{2}$ norm.

According to Proposition 4.5, for all $n \in \mathbb{N}^{*}$, there exists $C_{n}$ such that, for all $h>0$,

$$
\forall t \in[0, T], \quad\left|u(t)-u_{h}(t)\right|_{2} \leq C_{n} h .
$$

The constant $C_{n}$ is depending a priori on $n$. In order to study its dependence on $n$, let us use the results of Section 6; we can easily determine Lipschitz constant of each component of $f$ and we use then Proposition 6.3, which is more accurate than Proposition 6.1.

Let us set

$$
S_{1}(k)=\sum_{i=1}^{n} k_{i}, \quad S_{2}(k)=\sqrt{n \sum_{i=1}^{n} k_{i}^{2}} \text { and } S_{2}(\eta)=\sqrt{\frac{1}{n} \sum_{i=1}^{n} \eta_{i}^{2}} .
$$

According to the Cauchy-Schwarz inequality, we have

$$
\sum_{i=1}^{n} k_{i} u_{i} \leq \frac{1}{\sqrt{n}} S_{2}(k) \sqrt{\sum_{i=1}^{n} u_{i}^{2}}
$$

Let $q \in\{2,+\infty\}$; adopting notations (6.5) and (6.6), we have then

$$
\begin{aligned}
& L_{1}=0, \\
& L_{2}=\frac{1}{m}\left(k_{0}+\frac{1}{\sqrt{n}} S_{2}(k)\right), \text { if } q=2, \quad L_{2}=\frac{1}{m}\left(k_{0}+S_{1}(k)\right), \text { if } q=+\infty, \\
& L_{j}=1, \quad \forall j \in\{3, n+2\},
\end{aligned}
$$

and

$$
L= \begin{cases}\left(n+\frac{1}{m^{2}}\left(k_{0}+\frac{1}{\sqrt{n}} S_{2}(k)\right)^{2}\right)^{\frac{1}{2}}, & \text { if } q=2 \\ \max \left(1, \frac{1}{m}\left(k_{0}+S_{1}(k)\right)\right), & \text { if } q=+\infty\end{cases}
$$

Since $\left|u_{i, 0}\right| \leq \eta_{i}$, we have according to $(7.15)$

$$
\left\|f\left(., u_{0}\right)\right\|_{C^{0}\left([0, T], l_{n+2}^{q}\right)}= \begin{cases}\left(n y_{0}^{2}+\frac{1}{m^{2}}\left(\|F\|_{C^{0}([0, T])}+k_{0}\left|x_{0}\right|+S_{2}(k) S_{2}(\eta)\right)^{2}\right)^{\frac{1}{2}}, & \text { if } q=2, \\ \max \left(\left|y_{0}\right|, \frac{1}{m}\left(\|F\|_{C^{0}([0, T])}+k_{0}\left|x_{0}\right|+S_{2}(k) S_{2}(\eta)\right)\right), & \text { if } q=+\infty .\end{cases}
$$

Thus, assumptions (1.22), (1.24), (6.5) and (6.6) hold for $q \in\{2,+\infty\}$ and, according to Proposition 6.3, for all $n \in \mathbb{N}^{*}$, there exists $C_{n, q}$ such that

$$
\forall N \in \mathbb{N}^{*}, \quad\left\|u-u_{h}\right\|_{C^{0}\left([0, T], l_{n+2}^{q}\right)} \leq C_{n, q} h .
$$


Moreover, we may clarify expression of $C_{n, q}$ in terms of $n, q, u_{0}$ and $f$. Assume that there exists positive functions $\eta, k$ and $u_{0}$ in $L^{2}(0,1)$ such that

$$
\begin{aligned}
\forall i \in\{1, \ldots, n\}, & k_{i}=k(i / n) / n, \\
& \eta_{i}=\eta(i / n), \\
& u_{i, 0}=u_{0}(i / n) .
\end{aligned}
$$

We have

$$
\lim _{n \rightarrow+\infty} S_{1}(k)=\|k\|_{L^{1}(0,1)}, \quad \lim _{n \rightarrow+\infty} S_{2}(k)=\|k\|_{L^{2}(0,1)}, \quad \lim _{n \rightarrow+\infty} S_{2}(\eta)=\|\eta\|_{L^{2}(0,1)} .
$$

Thus, according to (7.12), (7.16) and (7.17), $\sup _{x \in \mathbb{R}^{n+2}}\|\partial f / \partial t(., x)\|, L$ and $\left\|f\left(., u_{0}\right)\right\|_{C^{0}\left([0, T], l_{n+2}^{q}\right)}$ are bounded uniformly in $n$ when $q=+\infty$; thus, according to (6.7), the constant $C_{n,+\infty}$ is bounded by a constant $C$ uniformly in $n$ such that

$$
\forall n \in \mathbb{N}^{*}, \quad \forall N \in \mathbb{N}^{*}, \quad\left\|u-u_{h}\right\|_{C^{0}\left([0, T], l_{n+2}^{+\infty}\right)} \leq C h .
$$

On the contrary, the expressions $L$ and $\left\|f\left(., u_{0}\right)\right\|_{C^{0}\left([0, T], l_{n+2}^{q}\right)}$ tend to infinity as $n$ tends to infinity with $q=2$ and we have not uniform estimates in $n$ of the constant $C_{n, 2}$.

Remark 7.1. The result proved by Lippold in [16] (see estimate (1.17)) is not valid here; indeed, we may write the system (7.8) and (7.9) under the form (1.8) and (1.9) with $B$ replaced by $C$, because $C$ is not positive. Indeed, if $x=(1,-1,0, \ldots, 0)$,

$$
\langle B(x), x\rangle=-k_{0} / m .
$$

\section{Numerical Simulations}

We choose for these numerical simulations functions $\eta, k$ and $u_{0}$ defined by

$$
\begin{aligned}
\forall s \in[0,1], & \eta(s)=s+0.1 \\
& k(s)=1 \\
& u_{0}(s)=0
\end{aligned}
$$

The values of $\left(\eta_{i}\right)_{i},\left(k_{i}\right)_{i}$ and $\left(u_{i, 0}\right)_{i}$ are defined by (7.19) and we choose

$$
m=1, \quad T=500, \quad k_{0}=x_{0}=\dot{x}_{0}=0, \quad F(t)=0.45 \cos (0.5 t) .
$$

One of the features of rheological models is the existence of hysteresis cycles; they are plotted in the $(x, F-m \ddot{x})$ plane, the second component being the reaction of the system (composed of springs and dry friction elements, without mass) to exterior forces. We did indeed observe these cycles, as reported in [1].

We discretized the system (7.8) and (7.9) by the numerical scheme (5.1) and (5.2) choosing

$$
N=1000000 \text { and } n \in\{3,10,100,700,1500\} .
$$

Five of the curves obtained are presented in Figures 3, 4 and 5.

We look secondly for an empirical order of convergence of the numerical scheme. We expect the error to be of the form

$$
\left\|u-u_{h}\right\|_{C^{0}\left([0, T], l_{n+2}^{q}\right)} \approx C_{n, q} h^{\alpha_{n, q}}
$$


TABLE 1. Values $\alpha_{n, q}, C_{n, q}$ and $r_{n, q}$ for different values of $n$, for $q=+\infty$ and $q=2$ for estimate (8.2).

\begin{tabular}{|c|c|l|l|l|}
\hline$q$ & $n$ & $\alpha_{n, q}$ & $C_{n, q}$ & $r_{n, q}$ \\
\hline \hline$+\infty$ & 3 & 1.00162 & 0.71734 & 0.999998 \\
\cline { 2 - 5 } & 10 & 1.00166 & 0.75184 & 0.999998 \\
\cline { 2 - 5 } & 100 & 1.00177 & 0.83276 & 0.999998 \\
\cline { 2 - 5 } & 700 & 1.00125 & 0.83106 & 0.999999 \\
\cline { 2 - 5 } & 1500 & 1.00164 & 0.83188 & 0.999998 \\
\hline \hline 2 & 3 & 1.00165 & 0.75175 & 0.999998 \\
\cline { 2 - 5 } & 10 & 1.00278 & 0.85304 & 0.999996 \\
\cline { 2 - 5 } & 100 & 1.00196 & 1.34671 & 0.999998 \\
\cline { 2 - 5 } & 700 & 1.00215 & 2.00740 & 0.999998 \\
\cline { 2 - 5 } & 1500 & 1.00215 & 2.36276 & 0.999998 \\
\hline
\end{tabular}

and we try to identify the numbers $C_{n, q}$ and $\alpha_{n, q}$. Define

$$
\varepsilon_{n, q}(h)=\left\|u_{h}-u_{h / 2}\right\|_{C^{0}\left([0, T], l_{n+2}^{q}\right)}
$$

then, formally

$$
\log \left(\varepsilon_{n, q}(h)\right) \approx \alpha_{n, q} \log (h)+\log \left(2 C_{n, q}\right) .
$$

A $\log$-log plot of $\varepsilon_{n, q}(h)$ versus $h$ gives an estimates of $C_{n, q}$ and $\alpha_{n, q}$.

We choose

$$
R=99, \quad h_{\min }=10^{-6}, \quad h_{\max }=10^{-2}, \quad q \in\{2,+\infty\}, \quad \forall j \in\{0, \ldots, R\}, \quad h_{j}=h_{\min }^{\frac{R-j}{R}} h_{\max }^{\frac{j}{R}},
$$

and the same physical parameters as above. Table 1 gives the values of $\alpha_{n, q}, C_{n, q}$ and the correlation of set of points $r_{n, q}$, versus different values of $n$.

We first see that the empirical order $\alpha_{n, q}$ and the correlation $r_{n, q}$ are close to one, which comforts estimates (7.18).

Otherwise, we can see in this table that the constant $C_{n,+\infty}$ seems to reach a limit value $C \approx 0.831$, what is coherent with the uniform estimate (7.20). However, we see that the constant $C_{n, 2}$ seems to increase without limit as $n$ increases.

More precisely, we can prove that if $k_{i}, \eta_{i}$ and $\eta_{i, 0}$ are defined by (7.19), then $S_{2}(k)$ and $S_{2}(\eta)$ defined at (7.14) are bounded independently of $n$ and we infer from (7.16) and (7.17) the equivalents

$$
\left\|f\left(., u_{0}\right)\right\|_{C^{0}\left([0, T], l_{n+2}^{2}\right)} \sim\left|y_{0}\right| \sqrt{n} \text { and } L \sim \sqrt{n} \text { as } n \text { tends to infinity. }
$$

Thus, according to (6.7), there exists $c$ such that

$$
\log \left(C_{n, 2}\right) \sim c \sqrt{n}
$$

Table 1 shows also that for $q=2$ and $n \in\{100,700,1500\}$,

$$
\frac{\log \left(C_{n, 2}\right)}{\sqrt{n}} \approx 0.02,
$$

what is again coherent with (8.3). 
(a)

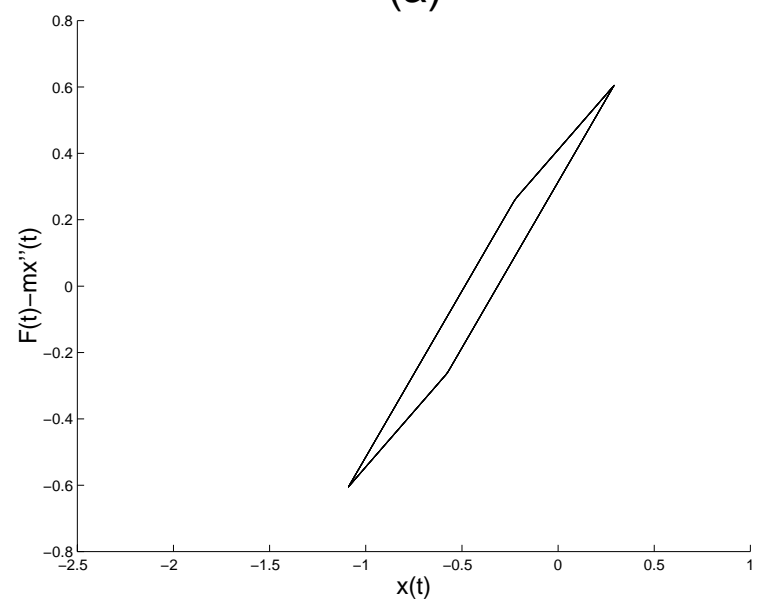

(b)

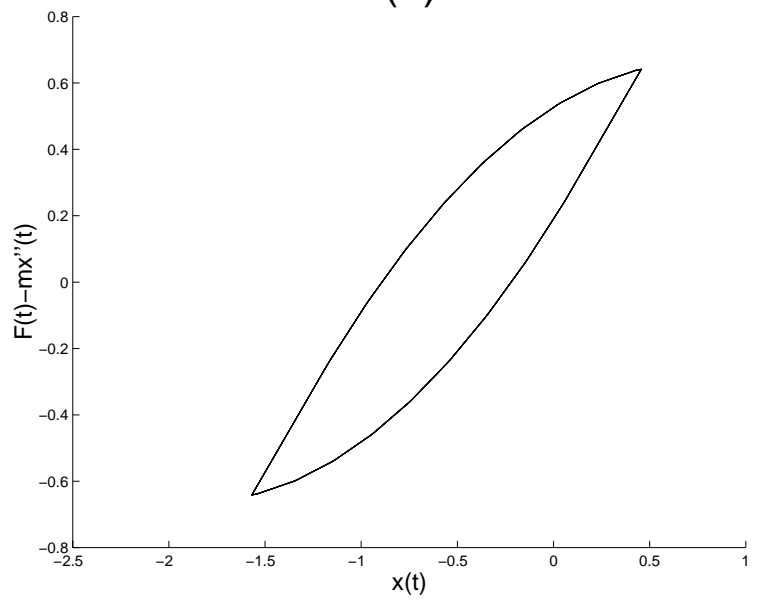

Figure 3. Curves $\{x(t), F(t)-m \ddot{x}(t)\}_{400 \leq t \leq 500}$ for $n=3$ (a) and $n=10$ (b).

(a)

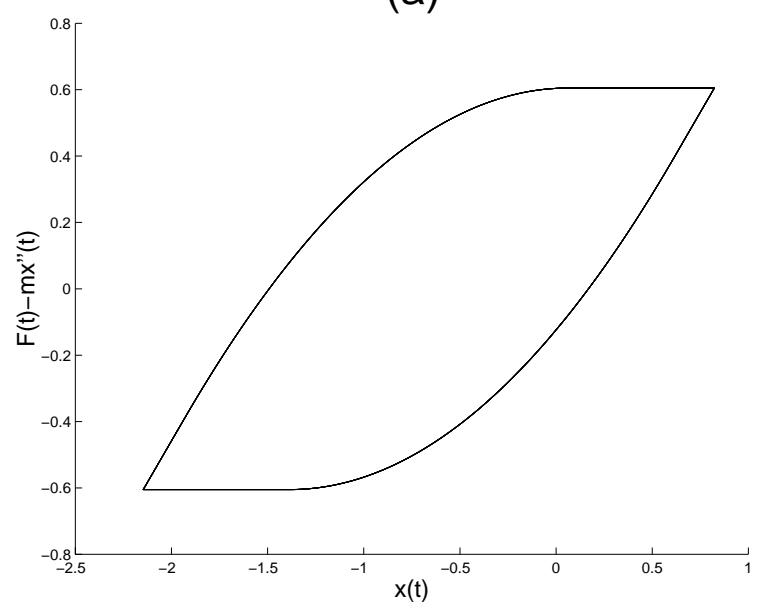

(b)

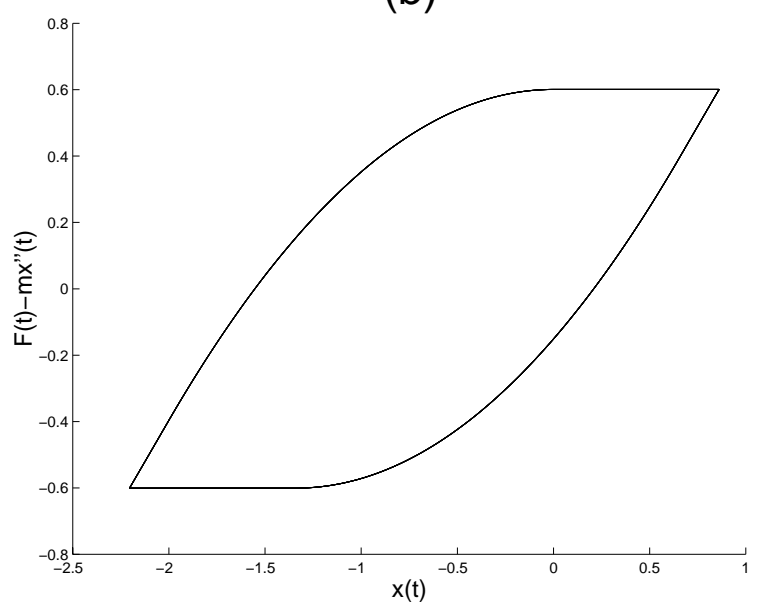

Figure 4. Curves $\{x(t), F(t)-m \ddot{x}(t)\}_{400 \leq t \leq 500}$ for $n=100$ (a) and $n=700$ (b).

\section{Conclusion And Perspectives}

In this paper, we extended the existence and uniqueness results of Brezis [5] to the differential inclusion (1.3) and (1.4) (its functional frame is more restricted but its form is more general).

We generalized Lippold's results of convergence [16] by proving the convergence of (1.5) and (1.6) to the solution of (1.3) and (1.4); this enables us to study the convergence of a numerical scheme adjusted to dynamical study of elastoplastic Prandtl model.

Figures 3,4 and 5 show that the hysteresis cycles obtained seem to tend to a limit cycle as $n$ tends to infinity. We will prove in a later work [2] that this limiting hysteresis cycle corresponds to the hysteresis cycle of a continuous Prandtl model with an infinite number of degrees of freedom. This model is defined by a spectrum of stiffness and threshold and it is equal to the limit of Prandtl model defined in Section 7 as $n$ tends to infinity. 


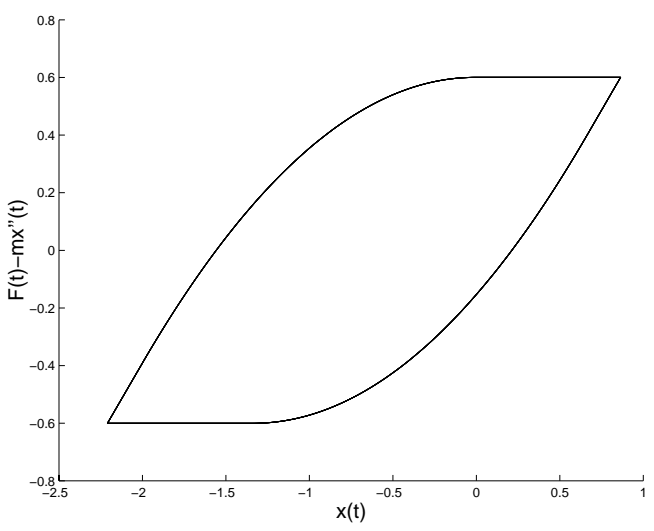

Figure 5. Curve $\{x(t), F(t)-m \ddot{x}(t)\}_{400 \leq t \leq 500}$ for $n=1500$.

\section{REFERENCES}

[1] J. Bastien, M. Schatzman and C.-H. Lamarque, Study of some rheological models with a finite number of degrees of freedom. Eur. J. Mech. A Solids 19 (2000) 277-307.

[2] J. Bastien, M. Schatzman and C.-H. Lamarque, Study of an elastoplastic model with an infinite number of internal degrees of freedom. Eur. J. Mech. A Solids 21 (2002) 199-222.

[3] J. Bastien, Étude théorique et numérique d'inclusions différentielles maximales monotones. Applications à des modèles élastoplastiques. Ph.D. Thesis, Université Lyon I (2000). number: 96-2000.

[4] H. Brezis, Perturbations non linéaires d'opérateurs maximaux monotones. C. R. Acad. Sci. Paris Sér. A-B 269 (1969) $566-569$.

[5] H. Brezis, Problèmes unilatéraux. J. Math. Pures Appl. 51 (1972) 1-168.

[6] H. Brezis, Opérateurs maximaux monotones et semi-groupes de contractions dans les espaces de Hilbert. North-Holland Publishing Co., Amsterdam (1973). North-Holland Mathematics Studies, No. 5. Notas de Matemática (50).

[7] M.G. Crandall and L.C. Evans, On the relation of the operator $\partial / \partial s+\partial / \partial \tau$ to evolution governed by accretive operators. Israel J. Math. 21 (1975) 261-278.

[8] R. Dautray and J.-L. Lions, Analyse mathématique et calcul numérique pour les sciences et les techniques. Vol. 8. Masson, Paris (1988). Évolution: semi-groupe, variationnel., Reprint of the edition of 1985.

[9] A.L. Dontchev and E.M. Farkhi, Error estimates for discretized differential inclusion. Computing 41 (1989) 349-358.

[10] A.L. Dontchev and F. Lempio, Difference methods for differential inclusions: a survey. SIAM Rev. 34 (1992) 263-294.

[11] M.A. Freedman, A random walk for the solution sought: remark on the difference scheme approach to nonlinear semigroups and evolution operators. Semigroup Forum 36 (1987) 117-126.

[12] U. Hornung, ADI-methods for nonlinear variational inequalities of evolution. Iterative solution of nonlinear systems of equations. Lecture Notes in Math. 953, Springer, Berlin-New York (1982) 138-148.

[13] A.G. Kartsatos, The existence of a method of lines for evolution equations involving maximal monotone operators and locally defined perturbations. Panamer. Math. J. 1 (1991) 17-27.

[14] F. Lempio and V. Veliov, Discrete approximations of differential inclusions. Bayreuth. Math. Schr. 54 (1998) 149-232.

[15] J.-L. Lions and E. Magenes, Problèmes aux limites non homogènes et applications. Vol. 1. Dunod, Paris (1968).

[16] G. Lippold, Error estimates for the implicit Euler approximation of an evolution inequality. Nonlinear Anal. 15 (1990) 10771089.

[17] V. Veliov, Second-order discrete approximation to linear differential inclusions. SIAM J. Numer. Anal. 29 (1992) $439-451$.

[18] E. Zeidler, Nonlinear functional analysis and its applications. II/B. Springer-Verlag, New York (1990). Nonlinear monotone operators, Translated from german by the author and Leo F. Boron. 